\title{
WFPC2 STUDIES OF THE CRAB NEBULA. I. HST AND ROSAT IMAGING OF THE SYNCHROTRON NEBULA ${ }^{1}$
}

\author{
J. Jeff Hester, ${ }^{2}$ Paul A. Scowen, ${ }^{2}$ Ravi Sankrit, ${ }^{2}$ Christopher J. Burrows, ${ }^{3}$ John S. Gallagher III, ${ }^{4}$ \\ Jon A. Holtzman, ${ }^{5}$ Alan Watson, ${ }^{5}$ John T. Trauger, ${ }^{6}$ Gilda E. Ballester, ${ }^{7}$ Stefano Casertano, ${ }^{8}$ \\ John T. Clarke, ${ }^{7}$ David Crisp, ${ }^{6}$ Robin W. Evans, ${ }^{6}$ Richard E. Griffiths, ${ }^{8}$ John G. Hoessel, ${ }^{4}$ \\ John Krist, ${ }^{3}$ Roger Lynds, ${ }^{9}$ Jeremy R. MOUld, ${ }^{10}$ EARL J. O’NeIL, JR., 9 \\ KARL R. STAPELFELdT, ${ }^{6}$ AND JAMES A. WeStPhaL ${ }^{11}$ \\ Received 1994 September 27; accepted 1995 January 31
}

\begin{abstract}
We present images of the Crab synchrotron nebula obtained with the Wide Field and Planetary Camera 2 (WFPC2) on board the Hubble Space Telescope. These data are compared with ROSAT HRI images, and with 0".5 resolution Canada-France-Hawaii Telescope (CFHT) images previously published by van den Bergh \& Pritchet (1989). These data strengthen the emerging picture of the Crab as a cylindrically symmetrical object with an axis running southeast to northwest, and inclined by $\sim 20^{\circ}-30^{\circ}$ with respect to the plane of the sky. Identification of structure very near to the pulsar which shares this symmetry helps to better establish the link between the symmetry axis of the nebula and the spin axis of the pulsar.

We report the discovery of a bright knot of visible emission located 0.65 to the southeast of the pulsar, along the axis of the system. This knot and a second knot 3".8 from the pulsar appear to be present but not well resolved in the $1988 \mathrm{CFHT}$ image, indicating that they are persistent structures. The inner knot is interpreted as a shock in the pulsar wind $\sim 1500 \mathrm{AU}$ above the pole of the pulsar. No corresponding knots are seen to the northwest of the pulsar, which may indicate that the characteristics of the wind from the two poles are not symmetrical. The closest of the "wisps" to the northwest of the pulsar appear to close into a ringlike "halo" encircling the axis of the nebula. The wisps are resolved, with widths of $\sim 0$ ". 2 . This allows calculation of their volumes and volume emissivities, and in turn their equipartition fields and pressures. Equipartition pressures calculated for the knots and wisps are typically 10 to as much as 80 times the equipartition pressure calculated for the nebula as a whole. The wisps show significant substructure which changed considerably between 1988 and 1994. Previous reports of relativistic motions of the wisps were probably due to changes in the unresolved substructure of these features. Comparison of the CFHT and WFPC2 images show remarkable changes in the inner nebula, but inferences about physical conditions based on this comparison are limited by the resolution of the CFHT data and the long 5 year baseline between the images. The structure of the nebula in 1994 may be inconsistent with the recent model by Gallant \& Arons (1994).

Very fine fibrous texture visible in the WFPC2 image follows the structure of the X-ray torus. A puzzling anticorrelation is seen between the X-ray and visible surface brightness through part of the torus. Long contiguous low contrast features with widths of $\sim 1^{\prime \prime}-2^{\prime \prime}$ are seen to run throughout the volume of the nebula. These features are seen to move outward through the nebula at velocities in excess of homologous expansion. These features trace the magnetic structure of the nebula; they are probably due to differences in emissivity accompanying varying degrees of departure from equipartition at roughly constant total pressure. Visible fibers "drape over" and appear to expand away from an X-ray counterjet to the northwest of the pulsar, supporting the interpretation of the counterjet as a directed flow of energy along the symmetry axis of the nebula. Other associations are also seen between X-ray and visible structures, including X-ray emission surrounding the east and west bays.

An association is proposed between the observed structure of the nebula and different latitude zones found in models of winds from partially oblique rotators. We concur with previous suggestions that the X-ray torus and the sharp visible fibers associated with it mark a shock at $r_{s} \sim 1.2 \times 10^{18} \mathrm{~cm}$ in an equatorial striped magnetic wind. The halo and anvil arise at a distance of $\sim 1.4 \times 10^{17} \mathrm{~cm}$ from the pulsar in a helically polar-
\end{abstract}

\footnotetext{
${ }^{1}$ Based on observations with the NASA/ESA Hubble Space Telescope, obtained at the Space Telescope Science Institute, which is operated by AURA, Inc., under NASA contract 5-26555.

${ }^{2}$ Department of Physics and Astronomy, Box 871504, Arizona State University, Tyler Mall, Tempe, AZ 85287-1504.

${ }^{3}$ Space Telescope Science Institute, 3700 San Martin Drive, Baltimore, MD 21218.

${ }^{4}$ Department of Astronomy, University of Wisconsin-Madison, 475 North Charter Street, Madison, WI 53706.

${ }^{5}$ Lowell Observatory, Mars Hill Road, Flagstaff, AZ 86001.

6 Jet Propulsion Laboratory, 4800 Oak Grove Drive, Pasadena, CA 91109.

7 Department of Atmospheric and Oceanic Sciences, University of Michigan, 2455 Hayward, Ann Arbor, MI 48109.
}

\footnotetext{
${ }^{8}$ Department of Astronomy, Johns Hopkins University, 3400 North Charles Street, Baltimore, MD 21218.

${ }^{9}$ Kitt Peak National Observatory, National Optical Astronomy Observatories, Box 26732, Tucson, AZ 85726. NOAO is operated by the Association of Universities for Research in Astronomy, Inc., under cooperative agreement with the National Science Foundation.

${ }^{10}$ Mount Stromlo and Siding Springs Observatories, Australian National University, Weston Creek Post Office, ACT 2611, Australia.

${ }^{11}$ Division of Geological and Planetary Sciences, California Institute of Technology, Pasadena, CA 91125.
} 
ized wind at latitudes greater than about $35^{\circ}$. Well collimated polar jets may be responsible for the knots to the southeast of the pulsar and for the jet and counterjet seen at X-ray and visible wavelengths.

Subject headings: ISM: individual (Crab Nebula) - ISM: jets and outflows - ISM: structure - X-rays: ISM

\section{INTRODUCTION}

The Crab Nebula is one of the most intensively studied objects in the sky. The body of work on the Crab is so voluminous that navigating the literature on the object is a feat in itself. (A recent SIMBAD dump of references for the Crab Nebula weighed in at a hefty 60 pages.) At the heart of the nebula is the Crab pulsar-the first pulsar with optically detected pulses (Cocke, Disney, \& Taylor 1969) - and an extensively studied source in all passbands from radio to $\gamma$-rays. Notable recent observations of the Crab pulsar include Compton Gamma Ray Observatory observations by Nolan et al. (1993) and visible and UV pulse-shape observations obtained with the Hubble Space Telescope high speed photometer by Percival et al. (1993). Nearer to the outer boundary of the nebula are the filaments - the chemically enriched material ejected by the explosion observed by Chinese astronomers in A.D. 1054 (e.g., review by Davidson \& Fesen 1985).

Tying together the pulsar and the filaments is the Crab synchrotron nebula: a relativistic magnetized plasma that is powered by the spin-down energy of the pulsar. The Crab Nebula was the first recognized astrophysical source of synchrotron radiation, as suggested by Shklovsky (1953) and confirmed by the polarization observations of Dombrovsky (1954). The conversion efficiency of the nebula is quite high, with $10 \%-20 \%$ of the spin-down energy released by the pulsar appearing as synchrotron radiation. Ultraviolet radiation from the synchrotron nebula ionizes the filaments (e.g., Henry \& MacAlpine 1982; Péquignot \& Dennefeld 1983), and the filaments in turn form a conducting cage that confines the synchrotron nebula (e.g., Michel et al. 1991).

The inner synchrotron nebula is a fascinating region consisting of jets, a torus of X-ray emission (e.g., Aschenbach \& Brinkmann 1975), small-scale variations in polarization and spectral index (e.g., Hickson \& van den Bergh 1990; Bietenholz \& Kronberg 1992), and complexes of sharp wisps (e.g., Scargle 1969). The rapid variability of the structure of the Crab has been known for some time (Lampland 1921; Oort \& Walraven 1956). The most comprehensive study of variability was that of Scargle (1969), who found that the wisps may show relativistic motions, but conversely always seem to be in more or less the same place. This structure is fascinating both in its own right and because of the opportunity that it presents for understanding the pulsar, its wind, and its interaction with the remnant of the explosion. Roughly $10 \%$ of the known supernova remnants are considered Crab-like, showing inner synchrotron emission assumed to be powered by pulsars. There are also a number of other nebulae known which are powered by the confined winds from pulsars (e.g., CTB 80, Hester \& Kulkarni 1988; PSR $1957+20$, Kulkarni \& Hester 1988; the "guitar nebula," Cordes, Romani, \& Lundgren 1993). Of these, however, the Crab is unique in the wide range of readily observable phenomena it displays.

Most theoretical discussion of the structure of the synchrotron nebula and its relationship to the pulsar starts with a paper by Rees \& Gunn (1974), who developed ideas originated by Piddington (1957) into a hydrodynamic model of the nebula. More recent treatments include those of Kennel \&
Coroniti (1984a, b), Coroniti (1990), Begelman \& Li (1992), and Michel (1994), all of which concentrate on understanding the relationship between a pulsar wind, which is expected to be dominated by field energy at its source (Michel 1982), and an observed flow which must be dominated by particle energy in order to shock and form the observed synchrotron nebula. These models generally associate the sharp wisps seen at visible and radio wavelengths with the location of the shock wave between the pulsar wind and the synchrotron nebula.

It has long been suspected that imaging investigations of the Crab Nebula were limited in a fundamental way by the spatial resolution of the data. This became clear when van den Bergh $\&$ Pritchet (1989) published a 0".5 resolution image of the Crab taken with the Canada-France-Hawaii Telescope (CFHT), revealing a wealth of subarcsecond structure. Even at 0".5, however, most of the structure in the nebula remained unresolved. We have undertaken a program to study the structure of the Crab Nebula at 0"1 resolution using the Wide Field and Planetary Camera 2 (WFPC2), which was recently installed in the Hubble Space Telescope. In this first paper (which follows a preliminary discussion by Scowen et al. 1994), we present images of the Crab synchrotron nebula taken through a relatively line-free filter centered near $5500 \AA$.

In principle, these data may represent a qualitative improvement over previous work, because they allow resolution of structures with a size comparable to the electron Larmor radius for electrons near the upper energy cutoff within the nebula. We discuss the WFPC 2 images of the Crab synchrotron nebula within the context of high signal to noise X-ray images of the Crab obtained with the ROSAT X-Ray Observatory, and begin a high-resolution study of the evolution of the Crab using the superb image obtained by van den Bergh \& Pritchet (1989). Subsequent papers will focus on the distribution of dust within the nebula and on the ionization structure of the filaments.

\section{OBSERVATIONS AND DATA REDUCTION}

\subsection{WFPC2 Imaging}

Observations of a field extending to the northwest of the Crab pulsar were obtained using the Hubble Space Telescope Wide Field and Planetary Camera 2. The WFPC2 is described in detail in the WFPC2 Instrument Handbook (Burrows et al. 1994) available through the Space Telescope Science Institute. The instrument has also been discussed by Trauger et al. (1994). Briefly, the WFPC2 is a reimaging camera that splits the focal plane of the telescope into four sections, then images each of these sections onto one of four CCDs. The CCDs are thick front-side-illuminated devices manufactured by Loral. They have a format of $800 \times 80015 \mu \mathrm{m}$ pixels. One of these "channels," referred to as the Planetary Camera, or "PC1," images the focal plane at a scale of $0.046 \mathrm{pixel}^{-1}$. The other three channels, referred to as the Wide Field Cameras, or "WF2-WF4," image the focal plane at a scale of $0.1 \mathrm{pixel}^{-1}$

Exposures were taken on 1994 March 9 through a relatively line-free continuum filter (F547M). The filter is a somewhat wider version of the Strömgren $y$ filter, with an effective central wavelength of $5480 \AA$, half power points at approximately 5150 
and $5820 \AA$, and a peak system response (throughput plus quantum efficiency) of $\sim 10.5 \%$. Two $1000 \mathrm{~s}$ integrations were taken to allow for cosmic-ray identification and removal. The data were recorded with the instrument in a gain state of 14 electrons per data number. The data were taken at the original CCD temperature setting of $-78^{\circ} \mathrm{C}$.

The calibration status of the WFPC2 as of this writing (summer 1994) is discussed by Holtzman et al. (1994). The data were corrected for slight differences in widths of the bins assigned by the analog-to-digital conversion in the instrument, then bias-subtracted using the average of the last seven columns of the overscan strip. Bias frames derived from stacks of on-orbit data were subtracted from each CCD. A difficulty with calibration of on-orbit WFPC2 data, especially at the warm $-78^{\circ} \mathrm{C}$ operating temperature, is keeping up with variations in hot and warm pixels that arise as a result of cosmicray-induced lattice dislocations in the CCDs. Dark subtraction of the data is a two-stage process. An average "superdark" frame was generated by taking the median of a large number of dark frames. Hot pixels were specifically excluded from this superdark. The superdark is subtracted from a smaller set of dark frames taken as close as possible to the science observations, which are used to identify hot pixels. These hot pixels are then scaled to the exposure time of a science frame and subtracted. In the case of the Crab F547M images, hot pixels were identified using a combination of five 2000 s dark frames taken on 1994 March 8.

Cosmic rays were removed from the frames by comparing the two images and looking for pixels in which the difference between the frames was greater than 4 times the expected $\sigma$ in the difference. Pixels adjacent to those detected in this way were then tested at the $2 \sigma$ level. Pixels in which one of the two images contained a cosmic ray were replaced by the other. Pixels in which both images were judged to contribute good data were assigned a value equal to the average of the two frames. While some coincidences in cosmic rays occurred between the two frames, this was not a severe problem.

The data were then flattened using flats that were generated from a combination of device- and filter-dependent data obtained during prelaunch system level thermal vacuum testing of the WFPC2 and a determination of the illumination pattern of the HST Optical Telescope Assembly derived from on-orbit exposures of the bright Earth. These are the same flats that are used to populate the STSCI WFPC2 Calibration Database. The flats are thought to be good to an rms level better than $1 \%$. The flats and their generation are discussed by Holtzman et al. (1994).

It was discovered on orbit by analysis of stellar photometry data that at an operating temperature of $-78^{\circ} \mathrm{C}$ the WFPC2 CCDs can show effective variations in sensitivity between the top and bottom of the device as a result of charge transfer inefficiencies arising from the presence of phosphorus vacancy traps in the silicon. The size of this effect can, under certain circumstances, exceed $10 \%$ peak to peak between the top and bottom of the CCD. (This effect is significantly less at lower temperatures. A combination of this fact and a desire to reduce the dark current from hot pixels motivated a decrease in operating temperature of the WFPC2 CCDs in 1994 mid-April.) Detailed discussion of this effect is beyond the scope of the current paper. However, both test data on the detectors and the physical model of the effect indicate that it should not strongly affect diffuse emission. Overall, we believe errors in relative photometry due to flat fielding errors and charge transfer efficiency (CTE) effects to be less than 3\%.
The images were combined into mosaics using a distortion mapping of the CCDs based on a fit to a radial cubic distortion model using the measured edges of the pyramid shadows present in the data. It should be stressed that this fit is not astrometrically accurate, but does provide a good enough match along the boundaries between adjacent CCDs to allow structure in the nebula to be followed across these boundaries and to allow comparison of the WFPC2 data with lower resolution ground-based data across the full field of view of the camera.

Figure 1 (Plate 10) shows the final mosaicked image. A square root stretch is used. The position angle of the columns in the figure is $\sim 26^{\circ}$ west of north, based on a comparison with the orientation of the PFUEI image presented in $\S 2.2$ below. The pulsar is located near the eastern edge of WF2, with the rest of the field covering a region in the northwestern quadrant of the nebula. (The pulsar was not centered in the PC, as would have been appropriate for a program devoted to a study of the synchrotron nebula, because these images were taken as part of a larger program also aimed at studies of the line emission from the filaments.) Figure 2 (Plate 11) shows a high-pass filtered version of the WFPC2 mosaic generated by subtracting a Gaussian-smoothed version of the image (kernel FWHM $=5^{\prime \prime}$ ) from the full resolution data.

\subsection{ROSAT HRI Images}

Soft X-ray images of the Crab taken with the High Resolution Imager (HRI) on the ROSAT X-Ray Observatory were obtained from the High-Energy Astrophysics Science Archive Research Center (HEASARC) ROSAT archives at Goddard Space Flight Center. These images were obtained and first presented by Aschenbach (1992). Photon event lists were extracted for eight exposures taken on 1992 September 16. The total exposure time in the sequence was $24,065 \mathrm{~s}$. The photon event lists were converted into images using the MAKE_IMAGE procedure contained in the HEASARC IDL ROSATLIB package. Each of the eight exposures had a slightly different pointing to minimize field-dependent effects on surface brightness sensitivity and point-spread function. The images were aligned with each other and co-added.

The Crab field showed a pronounced large-scale halo, likely due to some combination of scattering within the instrument and interstellar scattering between the Crab and Earth (cf. Mauche \& Gorenstein 1989). The level of this halo varied significantly across the outer parts of the nebula, making it difficult to display the data in a way that showed faint outlying structure. We were able to fit the shape of this scattering halo empirically with a two-component exponential with $1 / e$ scale lengths of $18^{\prime \prime}$ and $145^{\prime \prime}$. To subtract the scattering to first order, we convolved the ROSAT image with this empirical scattering function, then subtracted the convolved image from the original. While this background subtraction is not perfect, it significantly improved our ability to trace faint emission in the outer nebula.

For comparison with the X-ray data we present groundbased visible images of the Crab synchrotron nebula obtained using the wide-field prime focus universal extragalactic instrument (PFUEI) on the Palomar $1.5 \mathrm{~m}$ telescope. The wide field PFUEI is a reimaging camera that images a $16^{\prime} \times 16^{\prime}$ field onto an $800 \times 800$ Texas Instrument CCD at a resolution of 1 .2 pixel $^{-1}$ (see description in Hester \& Kulkarni 1989). The image shown is a $700 \mathrm{~s}$ exposure taken on the night of 1988 January 12 . The image was taken through a relatively line-free continuum filter with a width of $100 \AA$ and a central wave- 


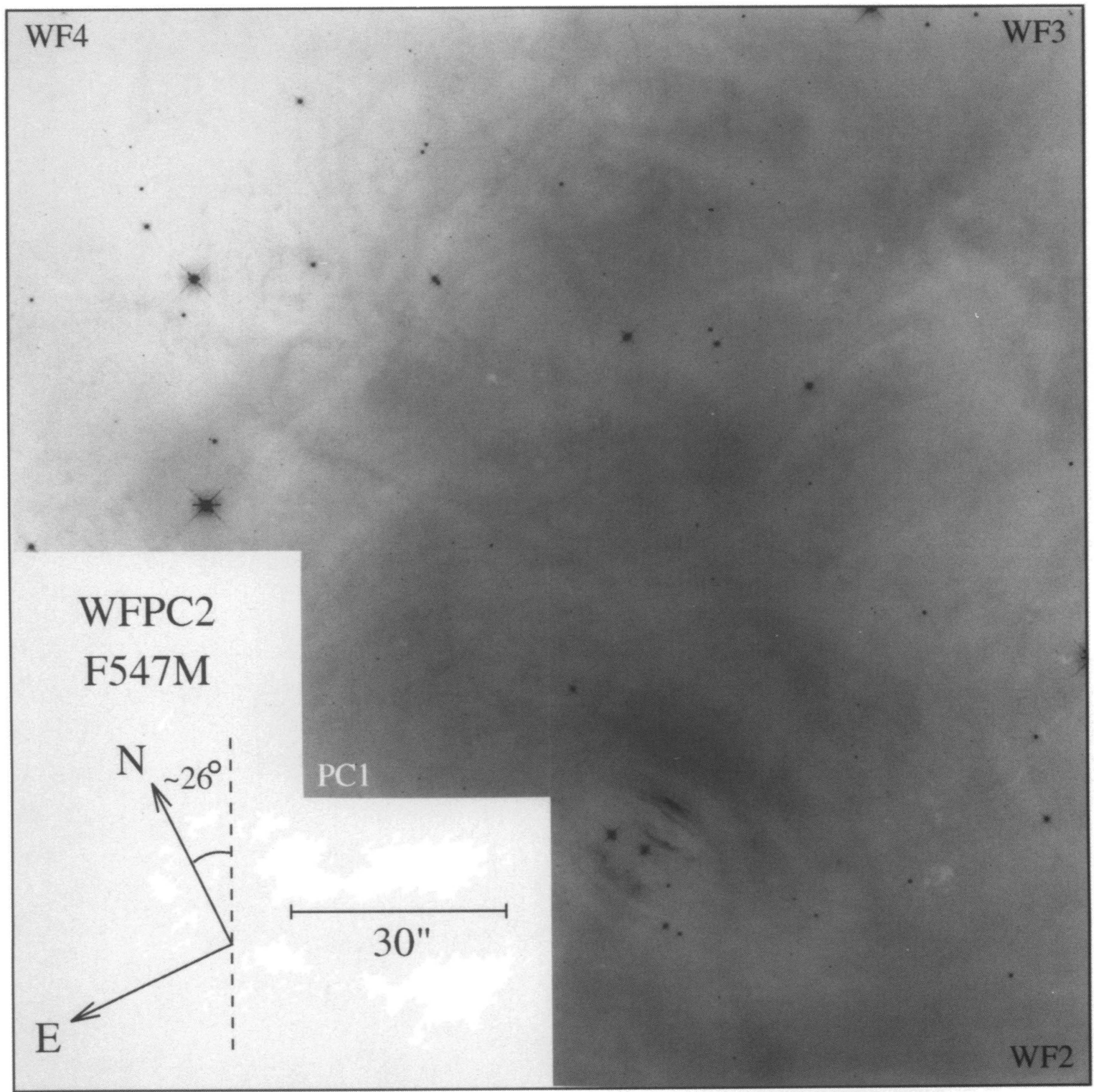

FIG. 1.-Mosaicked WFPC2 F547M image of the Crab Nebula. A square root stretch is used to improve dynamic range. The position angle of the columns in the figure is $\sim 26^{\circ}$ west of north. The field shown is 2.5 on a side. The length of the bar is $30^{\prime \prime}$. The identifications of the four cameras (PC1 and WF2-WF4) are shown in the corner of each quadrant. The pulsar is located near the eastern edge of the field of view of WF2.

Hester et al. (see 448, 242) 


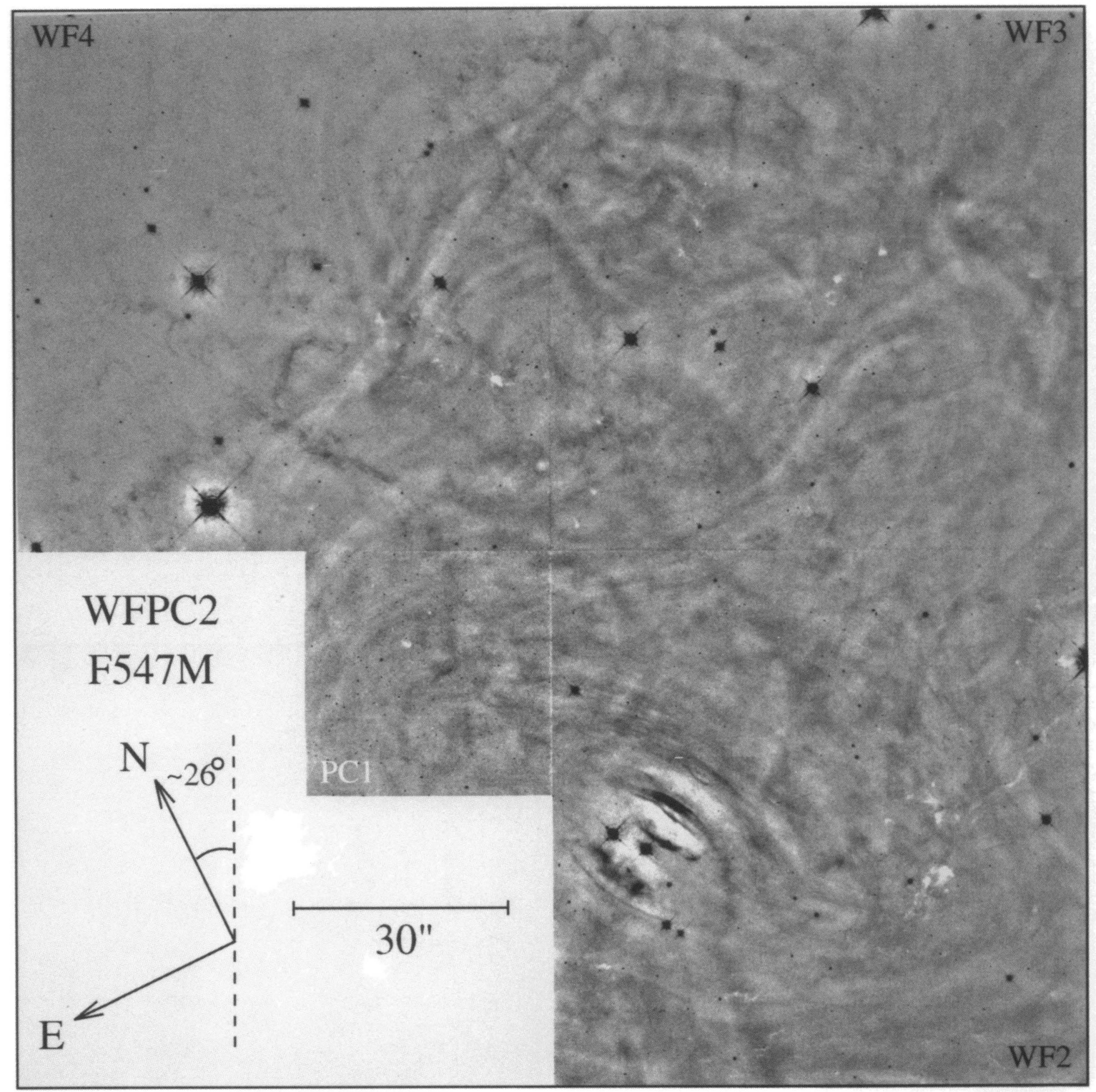

FIG. 2.-High-pass filtered version of the WFPC2 F547M mosaic of the Crab Nebula generated by subtracting a Gaussian filtered version of the image $\left(\mathrm{FWHM}=5^{\prime \prime}\right)$ from the original.

HeSTER et al. (see 448, 242) 
length of $5100 \AA$. The data were bias-subtracted using a single level derived from the on-chip overscan column, then flattened using dome flats. The central $6^{\prime} \times 6^{\prime}$ were extracted from the frame for comparison with the X-ray data. The 4 yr difference in epochs between the X-ray and visible light images is not ideally suited for detailed comparisons. However, while there have certainly been changes in detail in the structure of the nebula observed at visible wavelengths (see below), the general structure of the nebula (e.g., location of the bays, regions in which wisps are seen, and the appearance and extent of Baade's "underluminous zone") has remained fairly constant over decades. Thus, we feel fairly comfortable with making general qualitative comparisons between the X-ray and visible appearance of the nebula on the basis of these data.

Figure 3 (Plate 12) presents a comparison of the X-ray and optical data. Figure $3 a$ shows the $R O S A T$ image. The data are presented using a square root stretch to improve the dynamic range of the figure. Figure $3 b$ shows a high-pass filtered version of the X-ray image. This figure was generated by convolving a version of the data in which the pulsar had been suppressed with an 18" FWHM Gaussian kernel, then subtracting the result from the X-ray image. The result was convolved with a 3".6 FWHM Gaussian kernel. Figure $3 c$ presents the $5100 \AA$ image of the Crab, again with a square root stretch. Figure $3 d$
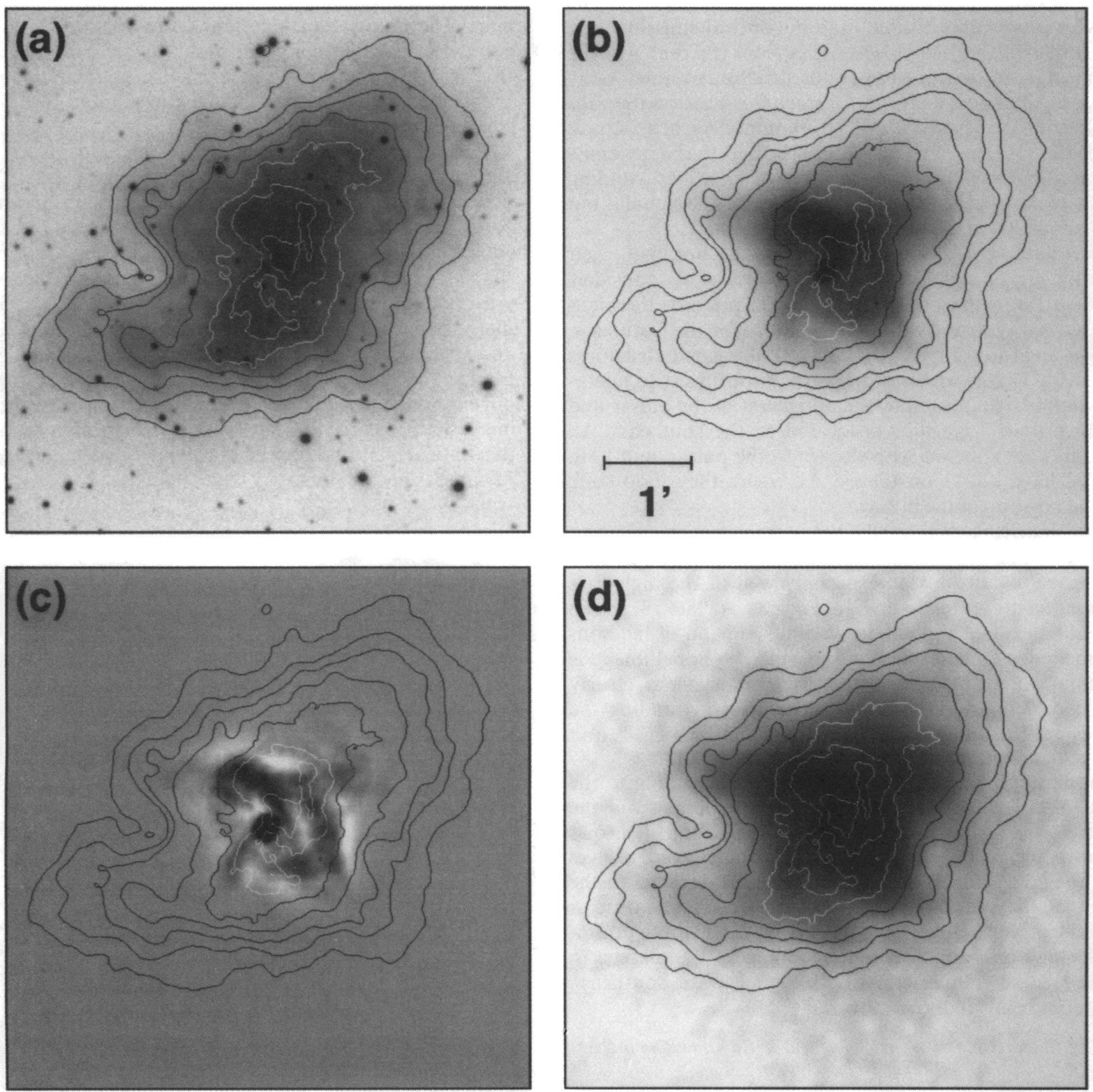

FIG. 4. - Comparison between the ground-based $5100 \AA$ data and the ROSAT X-ray images. (a) The $5100 \AA$ image with contours of itself overlaid. The contours are not uniformly spaced, but were specified by hand to generally trace the outline of structure apparent to the eye. The lowest four contours were generated using data that had been smoothed with a 7" FWHM Gaussian. The top three contours were generated using data that had been smoothed with a 2".5 Gaussian. Stars were suppressed prior to generating contours. The optical contours were then overlaid onto a number of representations of the ROSAT data. (b) ROSAT image, displayed with a square root stretch. (c) high-pass filtered image. (d) Deep logarithmically stretched image. 

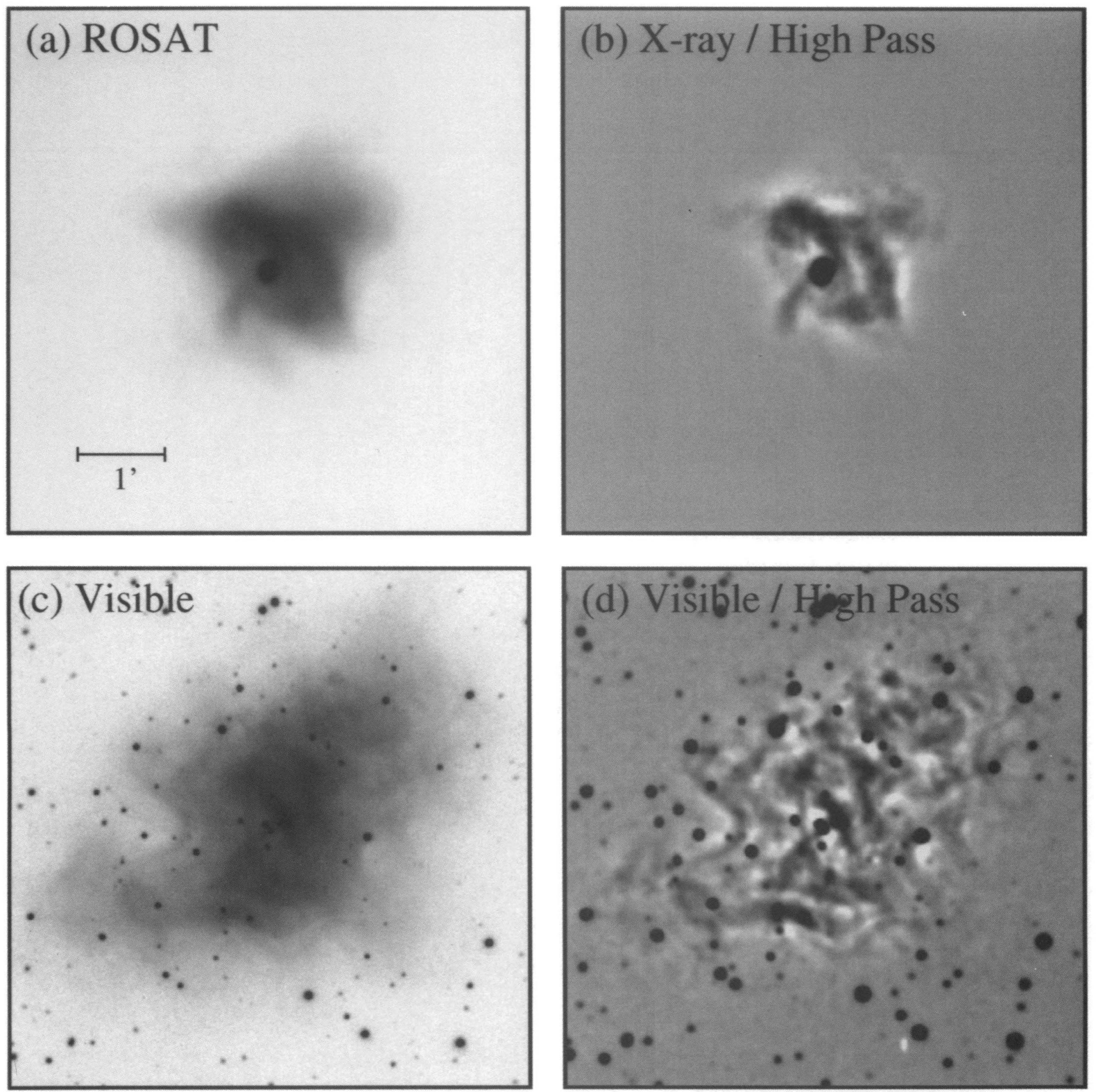

FIG. 3.-Comparison of the Crab Nebula at X-ray and visible wavelengths. (a) ROSAT HRI image of the Crab. The data are presented using a square root stretch to improve the dynamic range of the figure. North is at the top of the figure, and east is to the left. The size of the field shown is 6' square. (b) High-pass filtered version of the ROSAT image which shows the structure of features such as the X-ray torus, the jet, and the counterjet. The image was generated by convolving a version of the X-ray data in which the pulsar was suppressed with an $18^{\prime \prime}$ Gaussian, then subtracting the result from the original image. The result was then convolved with a 3.6 Gaussian to improve visibility of features. (c) A $5100 \AA$ image of the Crab obtained with the wide-field PFUEI on the Palomar $1.5 \mathrm{~m}$ telescope. The data are displayed with a square root stretch. $(d)$ The $5100 \AA$ image after high-pass filtering in the same way as the X-ray image in $(b)$. Stars were removed and replaced with the average of the surrounding emission prior to generating the smoothed frame which was subtracted from the original.

Hester et al. (see 448, 243) 
shows the visible light image after high-pass filtering the data in the same way as the X-ray image. Figure 4 shows a comparison between the ROSAT and the $5100 \AA$ images made by overlaying contours of the visible light image onto various representations of the X-ray data.

\section{RESULTS}

\subsection{The X-Ray Structure of the Crab}

We begin by considering the ROSAT image of the Crab, which provides a useful context for our more detailed consideration of the WFPC2 observations.

\subsubsection{The Inner Nebula}

From the earliest lunar occultation observations of the Crab (e.g., Bowyer et al. 1964) it was known that the X-rays from the nebula were more concentrated than the optical emission, and that the center of the X-rays was offset from the center of the visible object. Final clarification of this structure awaited X-ray observations obtained with the Einstein Observatory (Seward 1981; Harnden \& Seward 1984; Brinkmann, Aschenbach, \& Langmeier 1985). The Einstein images showed the X-ray emission from the Crab to be concentrated in a bar running southwest to northeast through the center of the nebula, but offset by about $30^{\prime \prime}$ to the northwest of the pulsar.

The new data generally confirm this picture but, with their significantly higher signal-to-noise ratio, also show considerable substructure within this bar. Figure $5 a$ presents the highpass filtered X-ray image with nomenclature for the features in this region. Features T1, T2, and T3 are elongated structures with widths of $\sim 20^{\prime \prime}$, which run generally southwest to northeast. T1 and T2 are located to the northwest of the pulsar and are brighter than T3, which is located to the southeast. All three features are concave with respect to the pulsar, and both $\mathrm{T} 1$ and T2 have hooks on their ends, where they loop back around the position of the pulsar.

As noted by Brinkmann et al. (1985), these features seem to close into a looplike structure which surrounds the pulsar. This loop of emission can be seen especially well in the high-pass filtered images in Figures $3 b$ and $4 c$. Figure $6 a$ (Plate 13) shows the X-ray image after applying a modified version of the nonlinear Sobel edge-enhancement algorithm. The Sobel image is of particular interest because its edge enhancement clearly shows a complete and continuous structure surrounding the pulsar, despite the difference in brightness among T1, T2, and T3 (cf. Fig. 2 of Brinkmann et al. 1985).

Also apparent in the images is a bright jet extending to the southeast of the pulsar. The jet appears to emerge from the pulsar at close to a right angle to the T1-T3 loop. It grows thicker with increasing distance from the pulsar and curves away to the south. There is another structure located to the northwest of the pulsar, opposite to the jet. This feature is fainter and less well defined than the jet, but is still quite prominent in the high-pass and Sobel-filtered images. We will refer to this feature as the counterjet. As with the jet to the southeast, the counterjet bends to the southwest.

\subsubsection{The Relationship between Visible and $X$-Ray Emission in the} Inner Nebula

There is a definite, albeit complex, relationship between the structure seen in the X-ray and visible images, including both correlations and anticorrelations between the two. There are numerous locations where prominent X-ray and/or optical features or edges correspond to features in the other band which may or may not be especially prominent. It is difficult to describe this relationship effectively in words.

Features along most of the lengths of $\mathrm{T} 1$ and $\mathrm{T} 2$ correlate with bright visible light features. At its southwestern end, T1 closely matches the visible light structure in the vicinity of the outer of Scargle's (1969) wisps (see Figs. $4 c$ and $4 d$ ). The northeastern end of T1 coincides with the northeast boundary of the plateau of visible emission to the north of the pulsar (Figs. $4 b$ and $4 c$ ). Likewise; T2 follows a band of optical emission along its northern extent. The band of lower surface brightness emission between $\mathrm{T} 1$ and $\mathrm{T} 2$ is fainter in visible light as well. The WFPC2 data also show a striated or fibrous texture which generally follows the X-ray structure associated with the torus (§ 3.3.3 below).

The X-ray jet coincides with a similar feature seen in visible light. The association between X-ray and visible light in the counterjet is more complex, and will be discussed in $\S 3.4 .9$. Briefly, while there is no clear visible counterjet, there are a number of visible features which run parallel to or along the edges of the counterjet.

The relationship between the $\mathrm{X}$-ray and visible emission along the southern portion and southwestern end of T2 is very different from that to the north of the pulsar. Here the arc of $\mathrm{X}$-ray emission follows a band of fainter than average visible emission that curls in toward the pulsar (part of Scargle's " $S$ shaped 'hole' of reduced intensity"). This can be seen in Figure 4 by examining the visible light contours that loop in toward the pulsar from the west. The anticorrelation between X-rays and visible emission at this location seems too well defined to be due to chance. The reality of this anticorrelation is supported by the fact that the fibrous texture seen in the WFPC2 images $(\S 3.3 .3)$ continues to follow $\mathrm{T} 2$ through this region. If this anticorrelation between $\mathrm{X}$-ray and visible emission is real, its cause is not obvious.

\subsubsection{Outer $X$-Ray Structure}

Figure $6 b$ shows a deep logarithmic display of the ROSAT image. This figure shows that the extent of the X-ray emission within the Crab is much greater than previously noted. In a number of locations, faint X-rays extend almost to the boundary of the synchrotron nebula as seen in visible light. Nomenclature for outer X-ray features is given in Figure $5 b$.

Features $\mathrm{OE}$ and $\mathrm{OSW}$ lie more or less along the direction of extension of the inner loop. OE extends into the protrusion in the nebula located to the north of the east bay, a well-known and persistent indentation in the periphery of the Crab (Fesen, Martin, \& Shull 1992 and references therein). Similarly, the $\mathrm{X}$-ray feature OSE extends into the region to the south of the east bay.

At very low signal most of the edge of the east bay can be traced as an escarpment in the brightness of the X-rays which is bounded by the well-defined rope of visible emission that outlines the east bay. This rope extends onto the north and south of the bay itself (Fig. 3d), where it serves as an outer boundary for the X-ray extensions OE and OSE. (Compare Figs. $3 d, 4 a$, and $4 d$.) In the case of OE, there is a loop of emission at its tip that overlies a similar loop formed by the visible rope.

In contrast to the east bay, the sharp outline of the west bay can be seen in X-rays around almost the entire roughly circular extent of the feature. X-rays extend into the regions of optical emission to the north and south of the bay, but in this case the southern extension is more prominent in X-rays. At a very low 

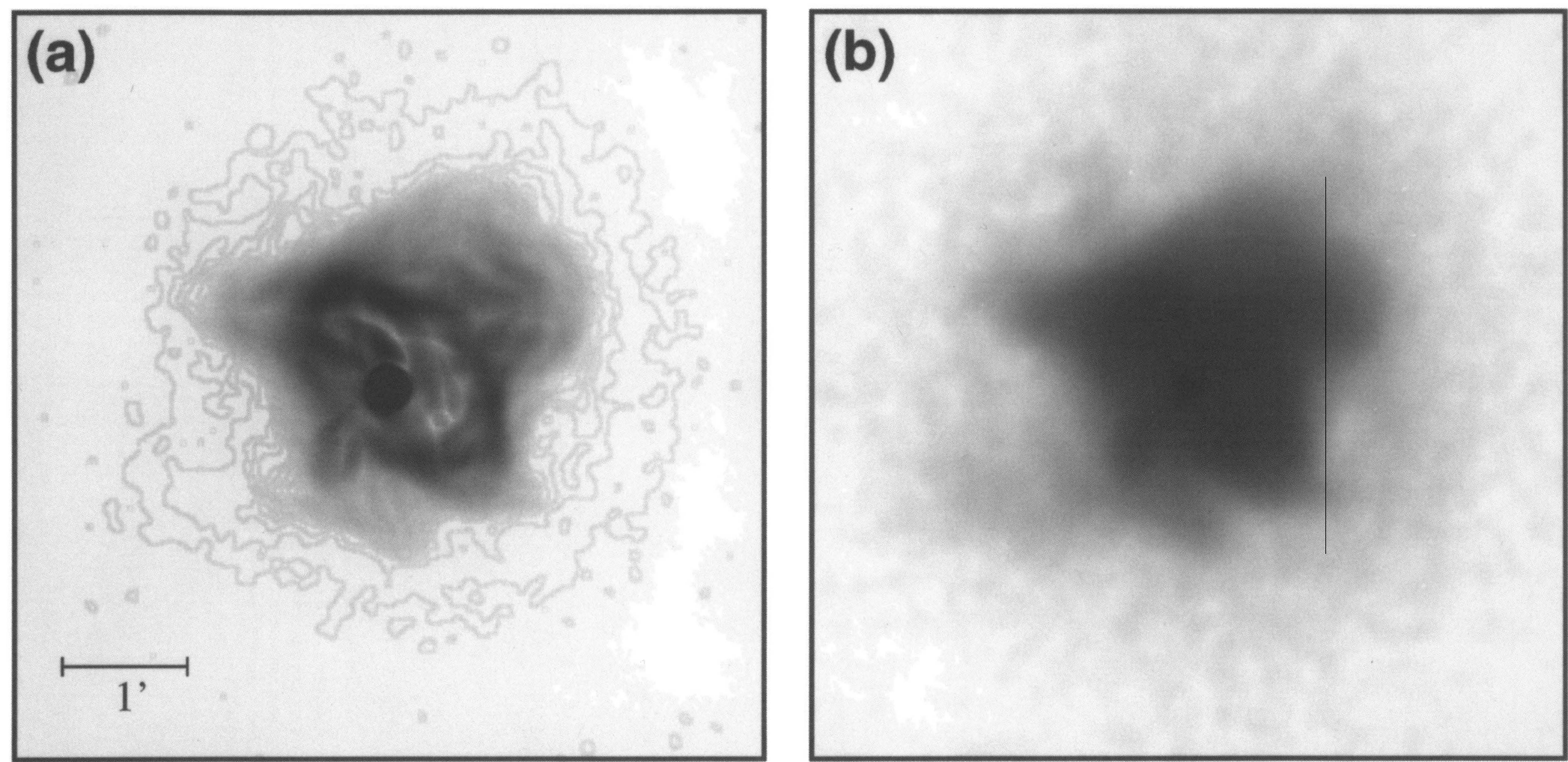

FIG. 6.-Two additional representations of the ROSAT images of the Crab Nebula. (a) ROSAT image filtered with the SOBEL edge enhancement algorithm. (b) Deep logarithmic stretch of the image showing the outer structure.

Hester et al. (see 448, 244) 

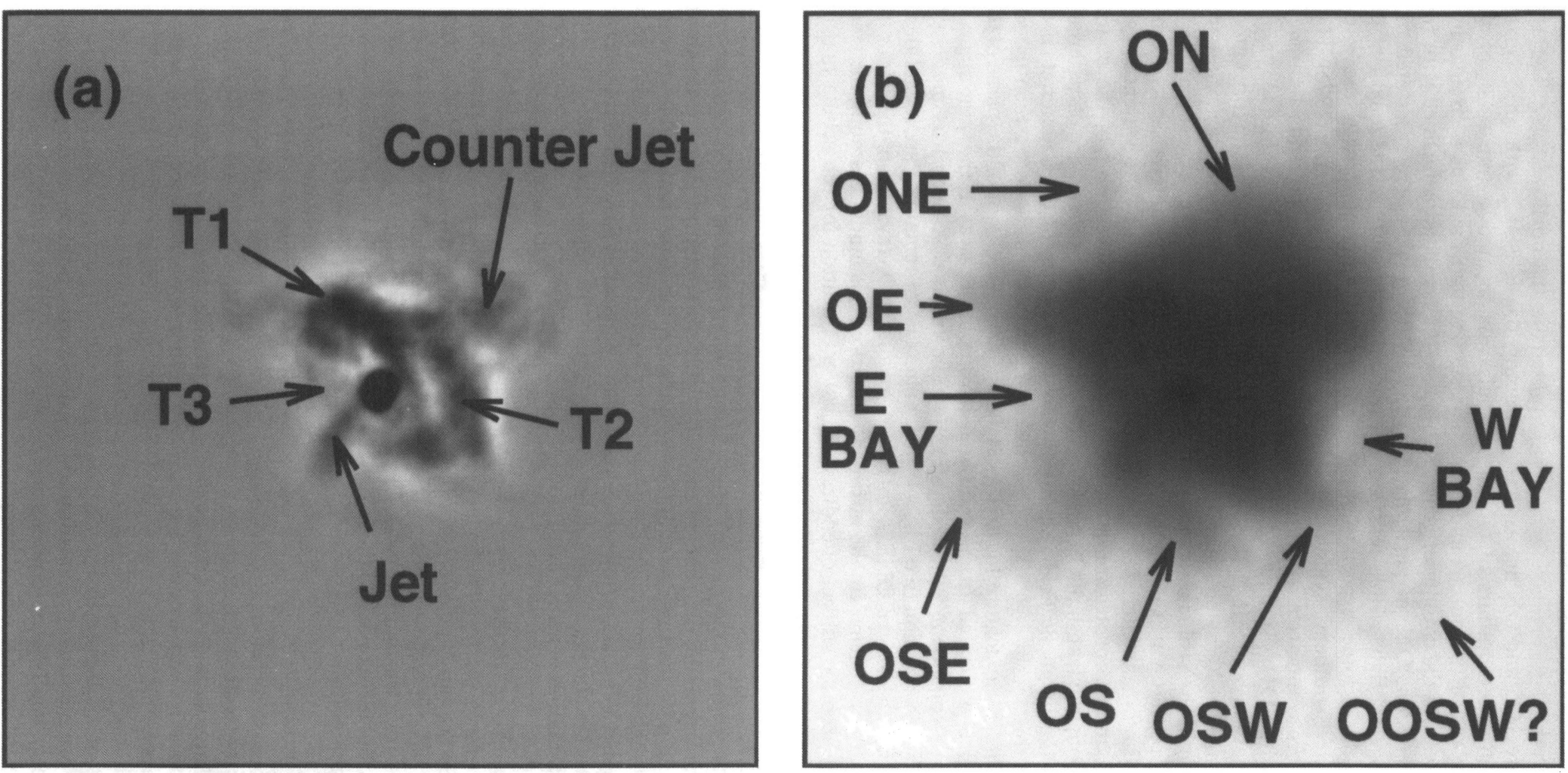

Fig. 5.-ROSAT image of the Crab with features identified for discussion. (a) High-pass filtered image of the Crab with portions of the X-ray torus (T1-T3) and the jet marked. (b) Deep logaritl stretch of the ROSAT image showing the faint outer structure of the nebula. 
signal level there appears to be an extension of the X-ray emission out as far as $\sim 3^{\prime}$ to the southwest of the pulsar (OOSW? in Fig. $5 b$ ). Faint $\mathrm{X}$-ray structure in this region follows the outermost visible contours in Figure 4.

As with most outer $\mathrm{X}$-ray features, $\mathrm{OS}$ and $\mathrm{ON}$ are associated with visible structures as well. The contours of ON (a large, fairly bright region to the north of the pulsar) and the diffuse emission to the south and west of the counterjet generally follow those of the visible emission. $\mathrm{ON}$, in particular, tucks in immediately behind the edge of bright visible emission along the northern edge of the nebula. In some respects, $\mathrm{ON}$ is the feature that breaks the northwest/southeast symmetry of the nebula, as can be seen in the more symmetrical appearance of the high-pass filtered images in which $\mathrm{ON}$ is suppressed.

An especially interesting association between X-rays and visible emission exists for OS, a finger of X-ray emission that pokes into a dimple-like protrusion in the visible synchrotron nebula. (This protrusion and the rope that marks its boundary can be seen especially well in the high-pass filtered image in Fig. $3 d$.) The relation between $O S$ and the protrusion is very suggestive that energy is flowing along the length of OS, and is being confined by the tension of the magnetic field running parallel to the edge of the nebula at this point.

Finally, we point out ONE, a faint X-ray feature located to the northeast of the pulsar. ONE is elongated from southsoutheast to north-northwest and is coincident with a slight protrusion in the contours of the visible emission (see Fig. 4d). It may or may not be significant that the axis of ONE points directly down the middle of the infamous "stem " on the north side of the Crab, a feature that has been the subject of endless speculation and controversy since its discovery by van den Bergh (1970). (The stem is most often referred to as the Crab's "jet"-terminology we avoid because of possible confusion with the X-ray and visible jet to the south of the pulsar.) This tubelike protrusion in the outer emission line filaments of the Crab (Gull \& Fesen 1982) is located near the top center of the field displayed in Figures 3-5. If elongated features in the outer $\mathrm{X}$-ray nebula trace conduits through which energy flows within the nebula, as may also be the case for the jet, the counterjet, and OS, then ONE may be a manifestation of a channeled flow of energy responsible for the stem (e.g., Bychkov 1975).

We should point out that not all X-ray features closely correspond to visible features. For example, the very hard edge in the bright X-ray emission along the western side of the nebula (see Figs. $3 a$ and $6 b$ ) has no obvious counterpart in the visible nebula but in fact cuts across a significant amount of visible structure. Likewise, there is a faint bubble-like cap to the X-ray counterjet that has no obvious visible counterpart.

\subsection{An X-Ray Torus and Jets}

Brinkmann et al. (1985) interpreted the elongated loop of $\mathrm{X}$-ray emission in the center of the Crab as confirming the earlier model of Aschenbach \& Brinkmann (1975), who proposed that the $\mathrm{X}$-ray emission from the $\mathrm{Crab}$ arises in a torus surrounding the pulsar. We find the ROSAT data to be consistent with this interpretation as well.

As previously noted, the emission to the northwest of the pulsar ( $\mathrm{T} 1$ and $\mathrm{T} 2$ ) is significantly brighter than the emission to the southeast (T3). This asymmetry has been interpreted by Pelling et al. (1987) as being due to Doppler upscattering of emission from material coming toward us. In this view, T1 and $\mathrm{T} 2$ are on the front of the torus, while T3 is on the back. The major axis of the torus is fairly well defined in the high-pass filtered image. It has a length of $\sim 83^{\prime \prime}$ and a position angle on the sky of $46^{\circ}$ east of north. The length of the minor axis of the torus is less well defined because of the significant substructure present in the northwest arc. The approximate length of the minor axis is $\sim 46^{\prime \prime}$, with an uncertainty of about $\pm 4^{\prime \prime}$. The pulsar is located within $\sim 3^{\prime \prime}$ of the center of the torus. Assuming that the torus is circular in shape, it has a radius of $1.2 \times 10^{18} \mathrm{~cm}$, and is inclined with respect to the plane of the sky by $33^{\circ} \pm 4^{\circ}$, where the stated uncertainty represents the quoted uncertainty in the length of the minor axis. This is in very good agreement with the angle of $30^{\circ}$ estimated by Aschenbach \& Brinkmann (1975). The thickness of the torus is $\sim 20^{\prime \prime}$, so the torus subtends an angle of $\sim 15^{\circ}$ as viewed from the pulsar. Again, this is close to the earlier result of $\sim 20^{\circ}$.

Within the context of the torus model, the jet and counterjet are naturally interpreted as being structures lying along the axis of the torus. We suggest that the jet and counterjet may be directed flows of material away from the pulsar. This view is supported in part by the presence of a dome or cap of emission over the northwest end of the counterjet. This cap has the morphological appearance of material which has been piled up by the advance of the counterjet. If the jet and counterjet lie along the axis of the torus, then the jet is coming toward us at an angle of $\sim 30^{\circ}$ out of the plane of the sky, while the counterjet is pointing away at the same angle.

The original X-ray torus model of Aschenbach \& Brinkmann (1975) remains a successful description of the morphology of the X-rays from the inner portion of the Crab. In addition, this model is among the evidence suggesting a physical organization for the nebula that is cylindrically symmetrical about an axis running from the southeast to the northwest through the position of the pulsar. We will find below that this physical picture provides a very useful context for discussion and interpretation of the detailed structure of the nebula at visible wavelengths. Aside from the interesting relationships between the $\mathrm{X}$-ray and visible emission from the Crab, per se, our inclusion of the ROSAT data in a paper that is predominantly about WFPC2 imaging was motivated by a desire to establish this physical picture.

\subsection{The Visible Structure of the Synchrotron Nebula at 0 ".1 Resolution}

\subsubsection{Knots}

We begin our discussion of the WFPC2 image of the Crab synchrotron nebula with what is perhaps our most obviously noteworthy result: the discovery of a bright knot of emission located $\sim 0$ " 65 to the southeast of the pulsar. This knot, along with a second similarly sharp but fainter knot located at a distance of 3 ."8 from the pulsar, lies at an approximate position angle of $115^{\circ}$ east of north. These knots are aligned with the $\mathrm{X}$-ray and optical jet to the southeast of the pulsar.

Both of the knots are elongated in the dimension roughly perpendicular to the jet direction, with lengths of $\sim 0$ ". 5 . To determine whether the knots were resolved, profiles were extracted across each knot and fitted with Gaussians. The widths of the knots measured in this way were compared with the similarly measured widths of star images in profiles taken at the same position angle. Both knots are resolved, with measured FWHM widths of 0 .'24 and 0 ".22, respectively, for the inner and outer knots. When combined in quadrature with the width of star profiles, the estimated true widths of the two knots are 0.16 and $0.12\left(4.8 \times 10^{15}\right.$ and $\left.3.6 \times 10^{15} \mathrm{~cm}\right)$, respectively. 
An enlargement of the immediate vicinity of the pulsar is shown in Figure $7 a$. To better isolate the knot from the pointspread function (PSF) of the pulsar, we scaled the star image to the northeast of the pulsar and subtracted it from the pulsar image. An image of the knot with the point source subtracted is presented in Figure $7 b$. Because of the need for a fractional pixel shift and the fact that both the star and the pulsar were saturated, and because of differences in spectrum between the two sources (and the effect this has on diffraction), the subtraction was not perfect. This could especially be seen in differences in the diffraction spikes of the two stars.

Despite the difficulties with the PSF subtraction, however,
Figure $7 b$ does show that the knot appears to be part of an arclike structure with a shape reminiscent of a bow shock. Figure $7 c$ shows this image with contours overlaid to better define the extent of the knot and arc. We are somewhat hesitant to claim that the fainter contours of the arc are real because they lie at about the same distance from the pulsar as a faint outer ring present in the PSF of bright stars in the field. However, with the caveat above concerning differences in diffraction, no plausible oversubtraction of the PSF from the pulsar makes this arc disappear. The arc itself is approximately $1^{\prime \prime}$ long, and subtends an angle of approximately $90^{\circ}$ with respect to the pulsar.
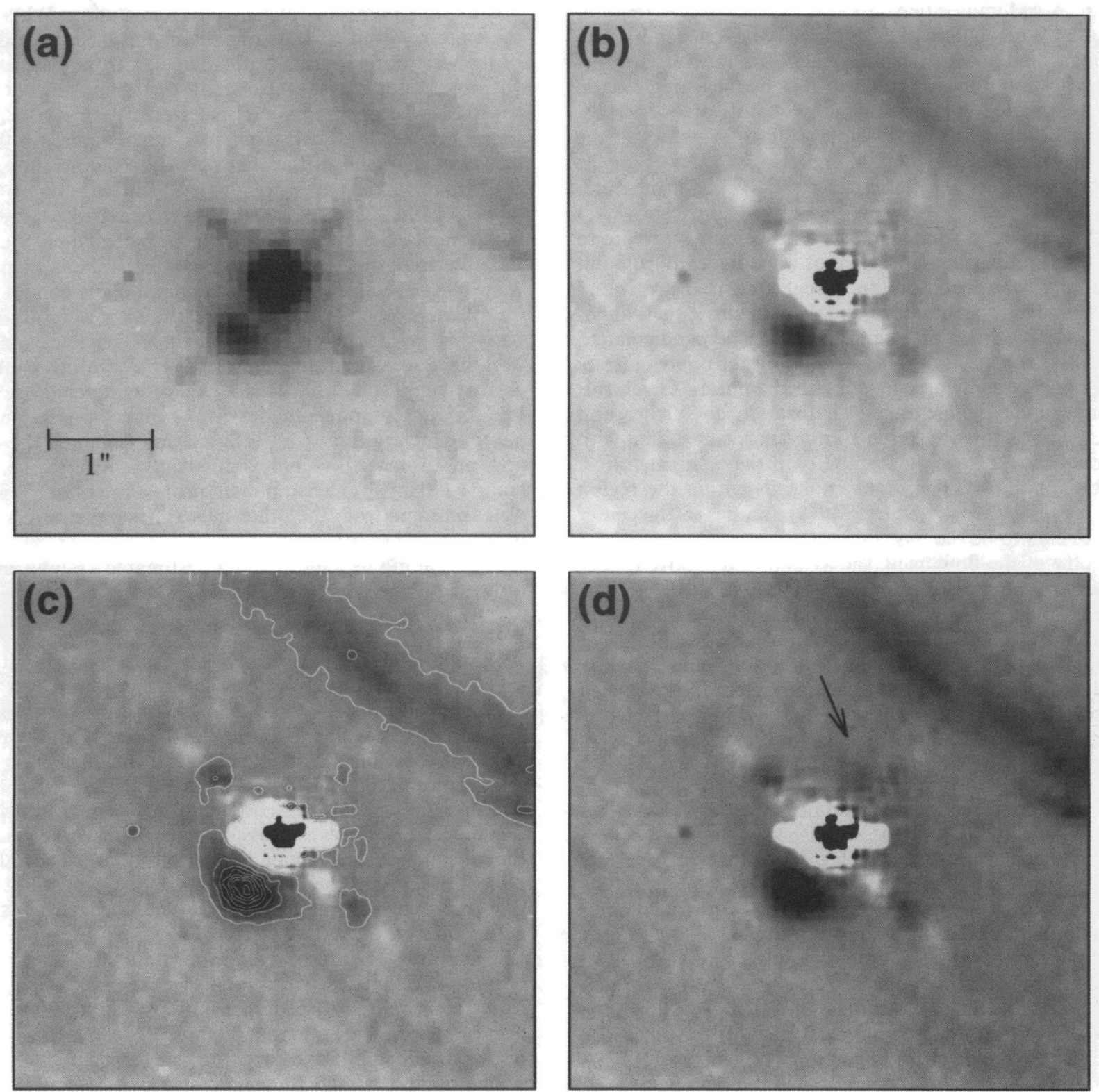

Fig. 7.-Blowups of the pulsar and the knot located 0.7 to the southeast of the pulsar. (a) Original image, displayed with a square root stretch. (b) Image after subtraction of a scaled and aligned version of the star immediately to the northeast of the pulsar. The subtraction is not perfect because of the need to resample the comparison PSF, differences in diffraction between the sources due to differences in their spectral shapes, and saturation in the two images. However, it does clearly show the knot and the arc within which it is embedded within. (c) PSF subtracted image with contours overlaid, showing the shape and extent of the knot. (d) PSF subtracted image, with a version of itself rotated by $180^{\circ}$, divided by 10 , and added to the original. This results in a fabricated "counterknot" (indicated by the arrow), which would be clearly visible were it present in the actual data. We conclude that any hypothetical counterknot would have to be fainter than the knot by at least a factor of 20 . 
The flux densities and luminosities of the knots were calculated using the values for the dimensionless efficiency $\int Q T d \lambda / \lambda$ of the HST + WFPC2 + F547M filter taken from the WFPC2 Instrument Handbook (Burrows et al. 1994), which have been found to agree with on-orbit calibration data to within $10 \%$ or so at this wavelength (Holtzman et al. 1994). Local backgrounds were extracted from the flux measured for each feature. The measured flux densities for knots 1 and 2 respectively are $2.16 \times 10^{-28}$ and $4.05 \times 10^{-29} \mathrm{ergs} \mathrm{cm}^{-2} \mathrm{~s}^{-1}$ $\mathrm{Hz}^{-1}$. Assuming a distance to the Crab of $2000 \mathrm{pc}$ and isotropic radiation (probably an invalid assumption), the luminosities of the two features are $1.03 \times 10^{17}$ and $1.94 \times 10^{16}$ ergs $\mathrm{s}^{-1} \mathrm{~Hz}^{-1}$.

To turn these luminosities into volume emissivities requires an estimate of the volume of each knot. We take each knot to be a squat cylinder with height equal to its measured transverse thickness, and radius equal to half its measured length. This gives estimated volumes for the two knots of $8.42 \times 10^{47}$ and $4.02 \times 10^{47} \mathrm{~cm}^{3}$, respectively, leading to estimates of the volume emissivities of $1.2 \times 10^{-31}$ ergs $\mathrm{cm}^{-3} \mathrm{~s}^{-1} \mathrm{~Hz}^{-1}$ for the inner knot and $4.8 \times 10^{-32} \mathrm{ergs} \mathrm{cm}^{-3} \mathrm{~s}^{-1} \mathrm{~Hz}^{-1}$ for the outer knot.

Since we have only a single image, and see only structure to the southeast of the pulsar, we cannot unequivocally rule out the possibility that the knots are foreground or background features. However, the uniqueness of these features, their alignment with the jet direction, their elongation perpendicular to the jet, and other aspects of their morphology strongly indicate that they are physically associated with the pulsar. Given this morphology and alignment, it is natural to go further and suggest that the knots (or at least knot 1) are associated with some sort of shock, wave, or instability in the collimated flow responsible for the jet. The outer knot falls within the region referred to as the "anvil" (Scargle 1969) and lies in the center of what appears to be the base of the visible jet. Correcting for the geometry of the flow using the assumptions above ( $\$ 3.2$ ), knot 1 lies at a physical distance of $\sim 1500 \mathrm{AU}\left(=2.3 \times 10^{16}\right.$ $\mathrm{cm}$ ) from the pulsar. The outer knot, knot 2, lies at a distance of $9060 \mathrm{AU}$, or $1.36 \times 10^{17} \mathrm{~cm}$ from the pulsar. Assuming that the jet is collimated to better than within a $90^{\circ}$ cone, the arc surrounding the inner knot and to a lesser extent the knot itself may represent material that has been diverted out of the jet by whatever wave or instability causes the knot.

\subsubsection{Emissivities of the Wisps}

The sharp arcuate features located near the pulsar were discussed by Scargle (1969), who established what has become standard nomenclature for these features. We will generally retain this nomenclature, and so begin by reviewing it briefly: Working outward from the pulsar to the northwest, the arc located at $\sim 1$." 8 is referred to as the "thin wisp." The next prominent feature, located 7".3 from the pulsar and visible here as an X-shaped merger of two structures, is "wisp 1." Scargle's "wisp 2 " is now the amorphous region located $\sim 14$ " from the pulsar. We will refer to the wisp located 8.3 to the southeast of the pulsar as the counterwisp. The "anvil" is the amorphous region of emission between the pulsar and the counterwisp. Identifications of the wisps, along with other features discussed in the text, are shown in Figure 8.

The WFPC2 data may in principle offer a qualitative improvement over previous studies of the Crab. Using canonical values for the field strength $(\sim 300 \mu \mathrm{G}$; e.g., Trimble 1982) and the high-energy cutoff of the electron spectrum inferred from the Crab's spectrum $\left(\gamma \approx 10^{9}\right.$; Rees \& Gunn 1974; Kennel \& Coroniti 1984b), the Larmor radius is $\sim 6 \times 10^{15}$ $\mathrm{cm} \approx 0$ ".2. This defines a natural size scale for structure within the nebula. While unresolved structure remains within the nebula even in the WFPC2 images, we do find that many features (e.g., the 0.2-0.3 transverse dimensions of Scargle's wisps) resolve out at just this scale. Because we are able to resolve these structures, we are able to estimate their volumes, which allows us to compute their volume emissivities. This is a key to learning their physical properties.

We begin closest to the pulsar with the "thin wisp" located 1".8 to the northwest of the pulsar. In addition to a smooth component with a thickness of $\sim 0^{\prime \prime} 37$, the thin wisp is seen to break into a number of sharp knots and clumps. While most of these are resolved, at least one filamentary feature located 2 ".2 to the west of the pulsar is not. The flux from a section of the wisp $\sim 3^{\prime \prime}$ long was measured, giving a flux density of $1.0 \times 10^{-28} \mathrm{ergs} \mathrm{cm}^{-2} \mathrm{~s}^{-1} \mathrm{~Hz}^{-1} \operatorname{arcsec}^{-1}$ along the filament. Assuming a cylindrical geometry with a radius of 0 "19 (the measured FWHM of the wisp) gives a volume emissivity for the wisp of $5.25 \times 10^{-32}$ ergs $\mathrm{cm}^{-3} \mathrm{~s}^{-1} \mathrm{~Hz}^{-1}$.

Wisp 1 (the 7".3 wisp) is seen here to break into three distinct components, the outer two of which are brighter. We refer to the outer and inner of these two components as wisp $1 \mathrm{~A}$ and wisp $1 \mathrm{~B}$, respectively. These two components come to within 0.3 of each other at their brightest points, where they essentially merge into a single bright wisp. The two components both have measured thicknesses of $\sim 0$ " 3 , with the outer component being somewhat thinner than the inner. Together, the two bright components of wisp 1 have a flux density of $4.9 \times 10^{-28} \mathrm{ergs} \mathrm{cm}^{-2} \mathrm{~s}^{-1} \mathrm{~Hz}^{-1} \operatorname{arcsec}^{-1}$ along their length, and an estimated volume emissivity $\epsilon_{v} \sim 5.8 \times 10^{-32}$ ergs $\mathrm{cm}^{-3} \mathrm{~s}^{-1} \mathrm{~Hz}^{-1}$. The component nearest the pulsar, wisp $1 \mathrm{C}$, is well separated from the other two. It has a measured width of 0.33 and a brightness of $7.5 \times 10^{-29}$ ergs $\mathrm{cm}^{-2} \mathrm{~s}^{-1} \mathrm{~Hz}^{-1}$ $\operatorname{arcsec}^{-1}$ along its length, and an estimated volume emissivity of $1.56 \times 10^{-32} \mathrm{ergs} \mathrm{cm}^{-3} \mathrm{~s}^{-1} \mathrm{~Hz}^{-1}$.

\subsubsection{The "Texture" of the Crab}

Scargle (1969) was possibly the first to note that images taken under conditions of excellent seeing hinted at delicate fibers embedded within the Crab. Figure 2 clearly shows the existence of these fibers, as well as the rest of the remarkable "texture" of the Crab synchrotron nebula. The general appearance of the high-pass filtered WFPC2 image is that of a painting in which the artist's long, sweeping brushstrokes remain, leading the eye across the face of the work. This texture runs throughout the nebula in a pattern that is generally reminiscent of maps of the polarization of the Crab, and it is likely that this texture is tracing the large-scale structure of the magnetic field.

The fibrous texture of the Crab is difficult to describe, both because of its intricacy and because of the confusion resulting from the projected three-dimensional structure of the nebula. Figure 9 shows the high-pass filtered WFPC2 image, with lines drawn to call attention to structures discussed in the text.

The highest spatial frequency structure is concentrated in the inner portions of the nebula. In particular, the vicinity of Scargle's wisp 2 no longer appears as a single wisp or even a small collection of well-defined or prominent wisps. Rather, this region now consists of more diffuse emission embedded within which are a dozen or more faint wisps. We refer to the sharpest of these as the T1 wisps because they lie along the T1 


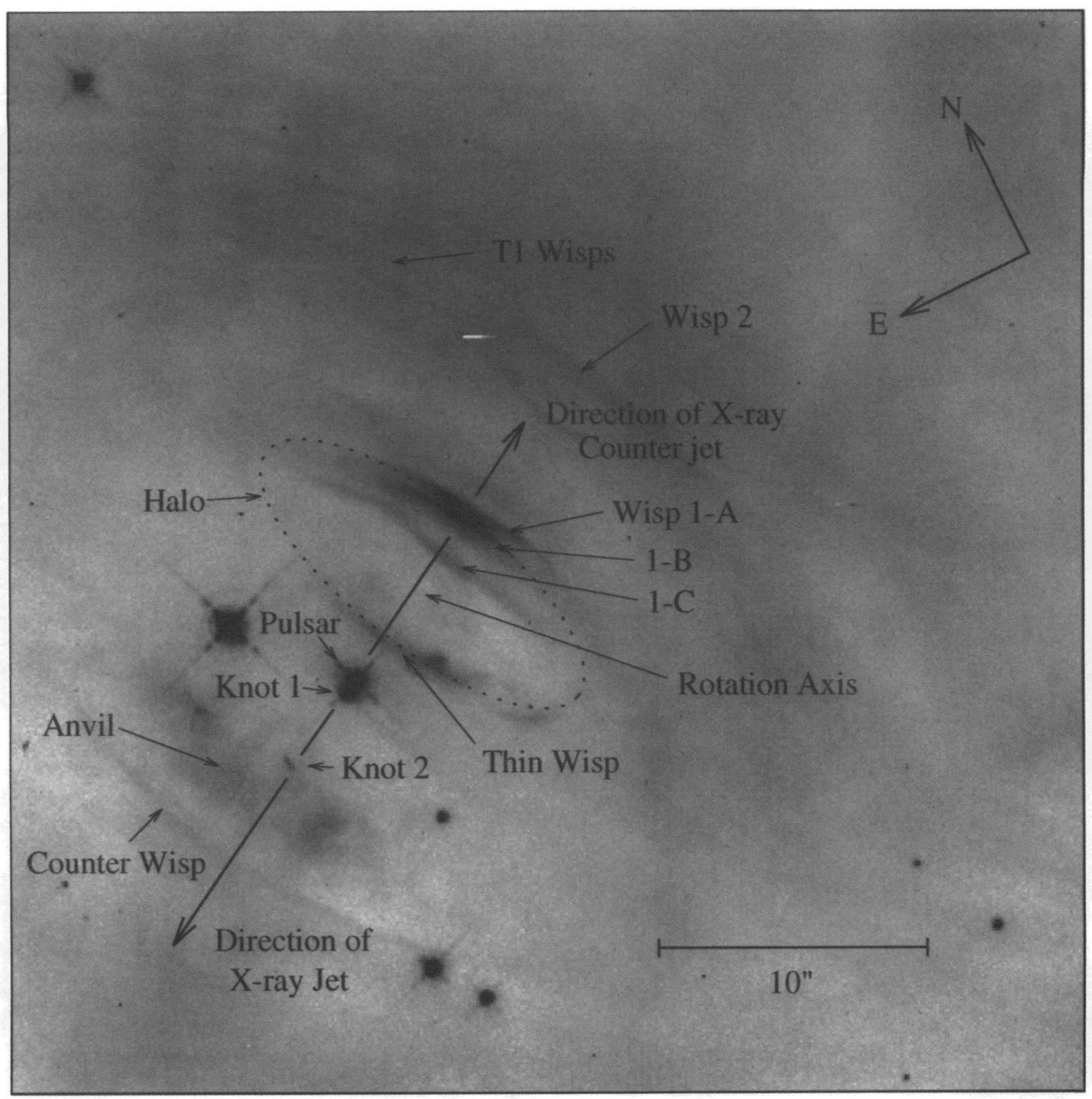

FIG. 8.-WFPC2 image of the vicinity of the Crab pulsar, with features labeled for discussion

portion of the $\mathrm{X}$-ray torus. These wisps have widths which range from the resolution limit to about 0.4 . The flux from these faint wisps is typically about $4.3 \times 10^{-30} \mathrm{ergs} \mathrm{cm}^{-2} \mathrm{~s}^{-1}$ $\mathrm{Hz}^{-1}$ above background per arcsecond along their length. This represents about a $\pm 3 \%$ modulation on the average brightness in this region. The rms variation due to such small scale structure is only about $2 \%$ of the local average. Assuming that the wisps are cylindrical with a diameter of 0 ". 25 , this gives a volume emissivity of $\sim 1.6 \times 10^{-33} \mathrm{ergs} \mathrm{cm}^{-3} \mathrm{~s}^{-1} \mathrm{~Hz}^{-1}$ for a typical "flux rope" in this region.

The T1 wisps must be embedded within or superposed on a region of emission that is truly more diffuse. To account for the smoothness of the emission in this region using an ensemble of wisps would require that an average line of sight cross about $(1 / 0.02)^{2}=2500$ wisps. Assuming a typical wisp diameter of $\sim 0$.'25 would then require a region that was at least 0.25 $\times 2500 \gtrsim 10^{\prime}$, which is significantly larger than the entire structure. We conclude that the majority of the emission even in this region of the Crab must come from a smoother component.

The relationship between the visible texture of the Crab and the X-ray structure is especially noteworthy. The T1 wisps are part of a "bundle" of fibers that follow the X-ray torus around the pulsar. This band contains the sharpest and most ordered visible texture in the nebula, apart from the bright wisps themselves. The usual explanation for the localization of the X-rays close to the center of the Crab is that the particles which give rise to the X-ray emission have short lifetimes. Hence the bright $\mathrm{X}$-rays trace the locus of particle injection into the nebula (i.e., the pulsar wind shock). The fact that very sharp visible texture is associated with the $\mathrm{X}$-ray torus then indicates that these sharp visible fibers are also associated with the wind 


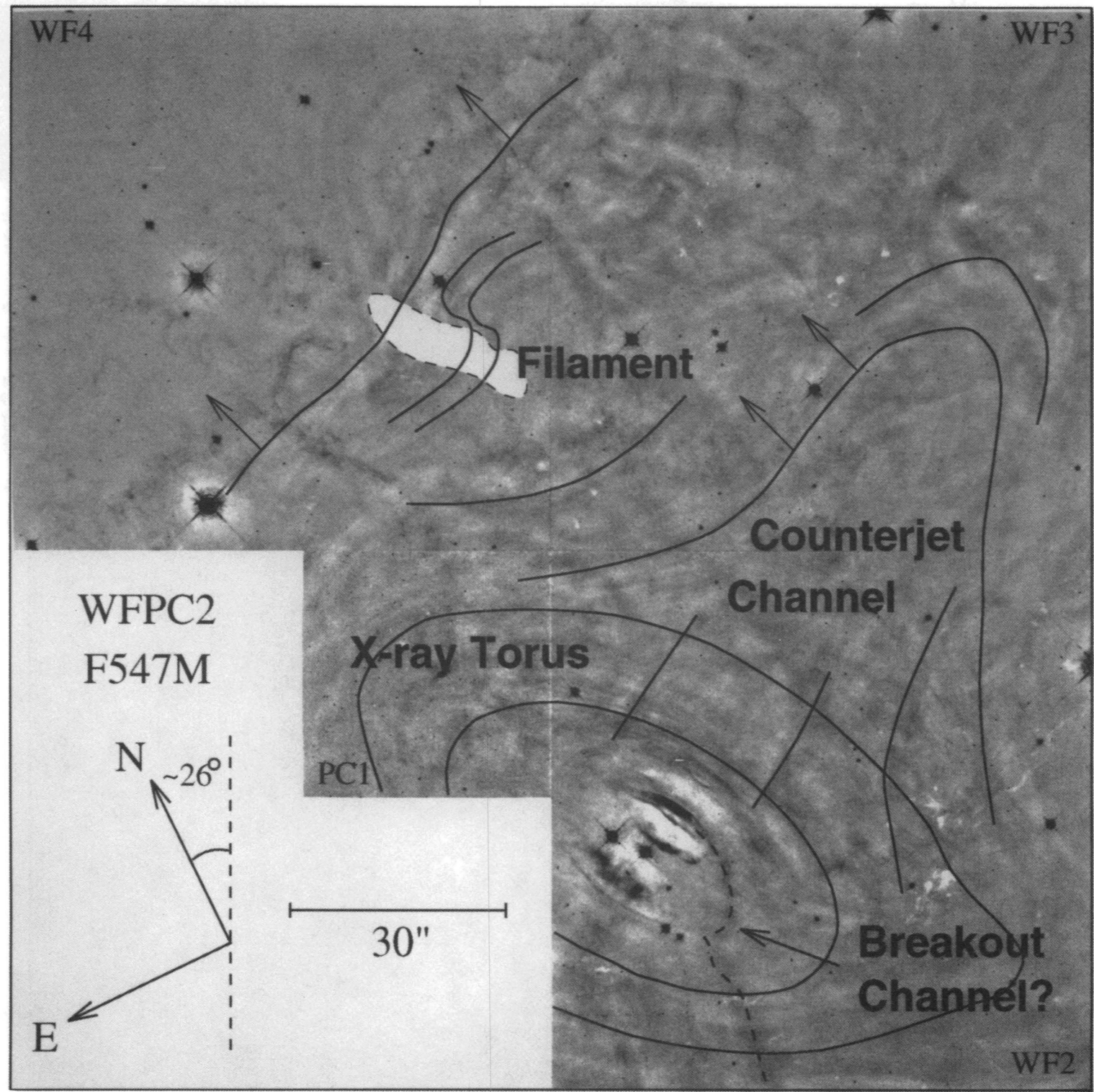

FIG. 9.-High-pass filtered WFPC2 image, with lines drawn in to indicate patterns in the "flow" of the fibrous or brushstroke texture of the nebula. These patterns are discussed in the text.

shock. If only a few of these visible fibers lie along a given line of sight, as argued above, then it is likely that these features are confined to a thin zone just outside the wind shock.

Farther away from the center of the nebula and regions associated with the $\mathrm{X}$-ray torus the visible texture of the nebula generally has a lower spatial frequency. In the outer parts of the nebula the long, continuous, "brushstrokes" still exist, but with transverse scales that are typically 1 1" -2 ".5. An especially interesting structure to the northwest of the pulsar is visible texture which parallels the counterjet, then loops over its top. Anticipating $\S 3.4 .9$ below, the region enclosed by this structure in the texture is labeled "counterjet channel" in Figure 9.

At least one clear interaction between the synchrotron nebula and the ejecta filaments can be seen in the field, near the southwestern edge of WF4 ("Filament" in Fig. 9). Here the texture of the synchrotron nebula wraps around the position of a broad filament. (The filament is traceable in Fig. 2 as a line of dust extinction features, and is clearly visible as residual line emission in the $I-V$ image presented in $\S 3.4$ below.) This is just the type of interaction between the magnetic structure of the synchrotron nebula and the cage of filaments described by Michel et al. (1991).

There are numerous other fascinating high spatial frequency structures present in the WFPC2 data. The reader with a serious interest in the magnetic topology of the Crab Nebula is encouraged to spend an enlightening few minutes examining Figure 2.

\subsubsection{Fields and Pressures}

Taking the average synchrotron emissivity of the Crab at $\sim 5500 \AA$ to be $\left\langle\epsilon_{v}\right\rangle \approx 2 \times 10^{-35}$ ergs s${ }^{-1} \mathrm{~cm}^{-3} \mathrm{~Hz}^{-1}$ (e.g., Craig et al. 1985), features such as the bright knot near the pulsar have emissivities that are as much as 6000 times the average of the nebula as a whole. These features must be regions within which the energy density is extremely high. 
To evaluate the departures from pressure equilibrium implied by the knots and wisps, we can in principle invert the synchrotron spectrum to obtain the field and particle parameters. In so doing, however, we encounter a number of ambiguities. For example, it is necessary to know the detailed spectral energy distribution of the emission. While the observed spectrum of the Crab in the visible and infrared is well approximated by a power law with index $\alpha=1.1\left(f_{v} \propto\right.$ $\left.v^{-\alpha}\right)$, when corrected for reddening the true spectral index may be as low as $\alpha=0.43$ (Wu 1981). This is a problem not only because of the uncertainty in $\alpha$ per se but also because for $\alpha<0.5$ the energy integral becomes strongly dependent on the maximum Lorentz factor, which is poorly known. The spectral index is known to change within the nebula, as do the values of the low- and high-energy cutoffs to the particle spectrum. The radiation from these features is also almost certainly not isotropic. Finally, the emissivity alone does not define both the magnetic field and the particle density. This requires a further assumption-normally equipartition between the particle and magnetic energy densities--which is also almost certainly violated (e.g., Kennel \& Coroniti 1984a; Begelman \& Li 1992).

Since it is not possible with information at hand to invert the emissivity properly, we proceed instead by inverting the emissivity conventionally. This will at least allow us to compare inferred conditions within the nebula according to a well understood set of assumptions. We begin with the assumption (Kennel \& Coroniti 1984b) that the radiation is mainly synchrotron radiation from a pair plasma. For a power law radiation spectrum of index $\alpha$, the particle population is also a power law with index $p=2 \alpha+1$, so $n(\gamma)=n_{0} \gamma^{-p}$. The volume emissivity of the plasma is given by

$$
\epsilon_{v} \equiv \frac{L_{v}}{V}=K(\alpha) B^{1+\alpha} v^{-\alpha} n_{0}
$$

where $K(\alpha)$ is a factor depending only on the spectral index (e.g., Pacholczyk 1970). Evaluating the energy integral for $p>2$ gives

$$
U=\int_{\gamma_{\min }}^{\gamma_{\max }} N(\gamma) E(\gamma) d \gamma=\frac{n_{0} m_{e} c^{2}}{2-p}\left(\gamma_{\max }^{2-p}-\gamma_{\min }^{2-p}\right),
$$

where $\gamma_{\max }$ and $\gamma_{\min }$ are the maximum and minimum Lorentz factors for the electrons (or positrons). Assuming equipartition between the field energy and particle energy gives

$$
n_{0} m_{e} c^{2}\left(\frac{\gamma_{\max }^{2-p}-\gamma_{\min }^{2-p}}{2-p}\right)=\frac{B^{2}}{8 \pi}
$$

Knowing the emissivity $\epsilon_{v}$, one can eliminate $n_{0}$ from the above equations and solve for the field strength $B$. This was done for two values of the photon spectral index, $\alpha=1.1$ and $\alpha=0.51$, for a suitable range of values of emissivity. We used the values $\gamma_{\min }=10^{4}$ and $\gamma_{\max }=10^{9}$ for the minimum and maximum Lorentz factors for the particles (e.g., Kennel \& Coroniti 1984b; Rees \& Gunn 1974). A plot of $\log B$ against $\log \epsilon_{v}$ is shown in Figure 10, together with emissivities of features discussed above.

It should be noted that while the exact values of the field and energy density calculated in this way depend somewhat on choice of spectral index and integration limits for the energy equation, relative comparison of conditions within the nebula does not. As long as the shape of the particle distribution remains the same throughout the nebula, which it probably does at least at the low energy end of the spectrum, which dominates the energy integral for $p>2$, then the equations above reduce to

$$
B \propto \epsilon_{v}^{1 /(3+\alpha)} \text { and } P \propto \epsilon_{v}^{2 /(3+\alpha)} .
$$

For $\alpha=1.1$ this gives $B \propto \epsilon_{v}^{0.24}$ and $P \propto \epsilon_{v}^{0.49}$. For $\alpha=0.51$ the results are not much different: $B \propto \epsilon_{v}^{0.28}$ and $P \propto \epsilon_{v}^{0.57}$. Using the standard value for the equipartition magnetic field of $3 \times 10^{-4} \mathrm{G}$ (e.g., Trimble 1982) and the value of $\left\langle\epsilon_{v}\right\rangle$ given

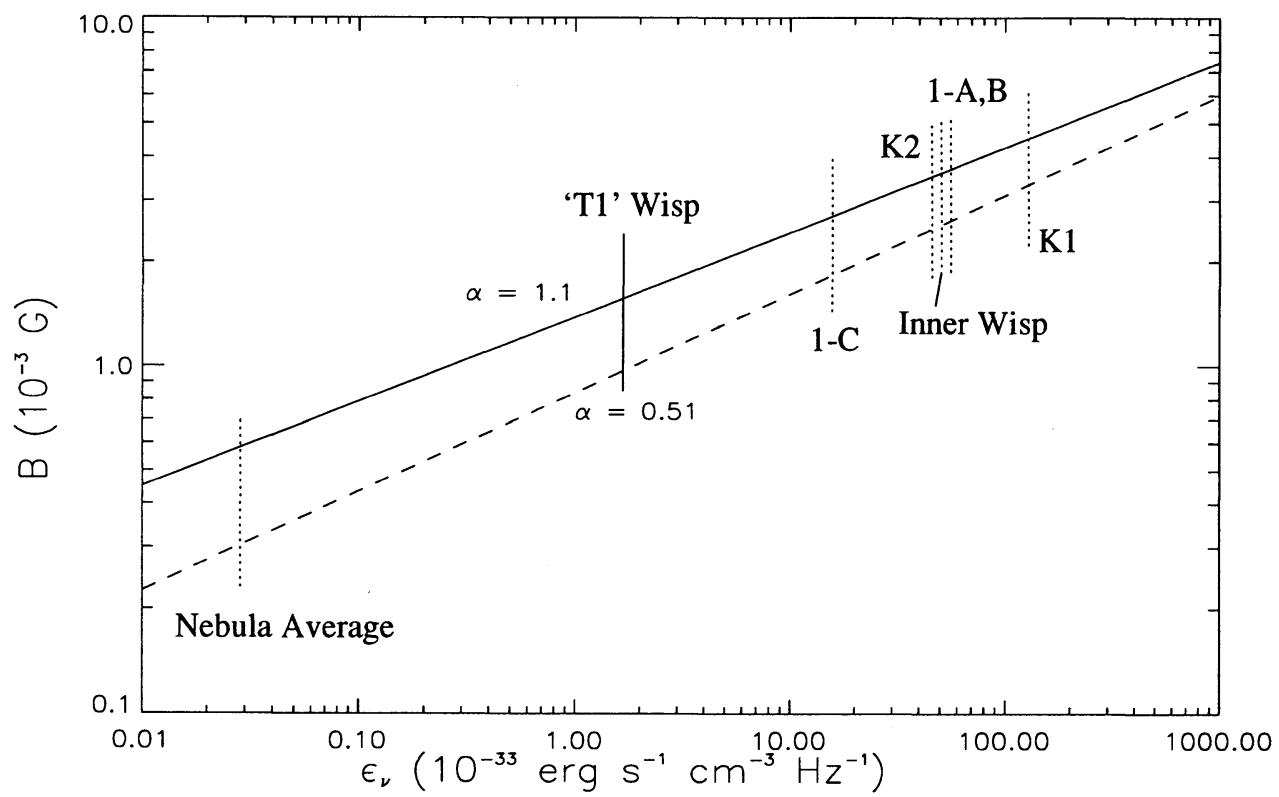

Fig. 10.-Plot of equipartition field strength vs. volume emissivity for two values of the spectral index. Also shown are the average volume emissivity of the Crab, as well as the emissivities of a number of sharp features in the nebula. 
TABLE 1

Crab Fluxes and Emissivities

\begin{tabular}{|c|c|c|c|c|c|c|}
\hline Feature & $f_{v}{ }^{\mathrm{a}}$ & $L_{v}^{\mathrm{b}}$ & Volume & $\epsilon_{v}=L_{v} / V^{\mathrm{c}}$ & $B_{\text {eq }}(\mu \mathrm{G})$ & $P /\left\langle P_{\text {neb }}\right\rangle$ \\
\hline Knot 1 & $2.16 \times 10^{-28}$ & $1.03 \times 10^{17}$ & $8.42 \times 10^{47}$ & $1.23 \times 10^{-31}$ & 2400 & 78 \\
\hline Knot $2 \ldots \ldots \ldots$ & $4.05 \times 10^{-29}$ & $1.94 \times 10^{16}$ & $4.02 \times 10^{47}$ & $4.83 \times 10^{-32}$ & 2100 & 49 \\
\hline Wisps $1 \mathrm{~A}+1 \mathrm{~B}^{\mathrm{d}}$. & $4.91 \times 10^{-28}$ & $2.35 \times 10^{17}$ & $4.07 \times 10^{48}$ & $5.79 \times 10^{-32}$ & 2200 & 54 \\
\hline Wisp $1 C^{d} \ldots \ldots$ & $7.46 \times 10^{-29}$ & $3.58 \times 10^{16}$ & $2.29 \times 10^{48}$ & $1.56 \times 10^{-32}$ & 1600 & 28 \\
\hline Thin wisp ${ }^{d} \ldots \ldots \ldots \ldots$ & $1.02 \times 10^{-28}$ & $4.88 \times 10^{16}$ & $9.27 \times 10^{47}$ & $5.25 \times 10^{-32}$ & 2100 & 51 \\
\hline "T1" wisp ${ }^{d} \ldots \ldots \ldots \ldots$ & $4.32 \times 10^{-30}$ & $2.07 \times 10^{15}$ & $1.32 \times 10^{48}$ & $1.6 \times 10^{-33}$ & 900 & 9 \\
\hline
\end{tabular}

${ }^{a}$ ergs $\mathrm{cm}^{-2} \mathrm{~s}^{-1} \mathrm{~Hz}^{-1}$.

${ }^{b}$ ergs s $^{-1} \mathrm{~Hz}^{-1}$.

c ergs $\mathrm{cm}^{-3} \mathrm{~s}^{-1} \mathrm{~Hz}^{-1}$

d For wisplike features, fluxes, luminosities, and volumes are quoted per arcsecond of wisp length, or per $3 \times 10^{16}$ cm of wisp length.

above, we adopt

$$
B=3 \times 10^{-4}\left(\frac{\epsilon_{v}}{2 \times 10^{-35}}\right)^{0.25} \mathrm{G}
$$

and

$$
P=\left(\frac{\epsilon_{v}}{2 \times 10^{-35}}\right)^{0.5}\left\langle P_{\mathrm{neb}}\right\rangle .
$$

The results of these calculations for a suite of features in the $\mathrm{Crab}$ are presented in Table 1 . Not surprisingly, since many of these features have small volumes yet surface brightnesses comparable to or greater than the average surface brightness of the nebula, the calculated fields and pressures in these features are very high. Scargle's wisps have equipartition fields ranging from about 900 to $2200 \mu \mathrm{G}$, which gives equipartition pressures from $\sim 10$ to 50 times the average pressure in the nebula. Conditions inferred for knot 1 are even more extreme, with an inferred field of $2400 \mu \mathrm{G}$, giving an equipartition pressure of almost 80 times the average equipartition pressure for the nebula.

This section began with a number of caveats concerning the certainty with which the synchrotron spectrum of the Crab can be inverted. Some models of the structure of the Crab find that the inner portion of the nebula is well out of equipartition, with $P_{B} / P_{\text {particle }} \lesssim 0.1$ (Kennel \& Coroniti 1984a; Begelman \& Li 1992). However, this only raises the pressure required to account for the wisp emissivities. Since $\epsilon_{v} \propto B^{1+\alpha} n_{0}$, then for $\alpha \sim 1$ the emissivity goes as $\epsilon_{v} \propto U_{B} U_{T}$, where $U_{B}$ and $U_{T}$ are the magnetic and particle energy densities, respectively. Thus, the minimum total energy density required to account for a given $\epsilon_{v}$ is the equipartition value, where $U_{B}=U_{T}$. We further note that since the field and pressure depend on the emissivity to low powers, the filament properties derived are not especially sensitive to uncertainties in filament volume or anisotropic radiation. For example, a factor of 2 error in determining the thickness of a wisp (a factor of 4 error in its volume) leads to only a factor of 2 error in the pressure inferred. Likewise, a factor of 2 error in determining the luminosity of a wisp due to anisotropic radiation leads to only a factor of $\sqrt{2}$ error in the pressure. On this basis, we suggest that our general conclusion-that the knots and wisps have very strong fields and pressures with respect to the rest of the nebula - is robust to the earlier caveats.

$$
\text { 3.3.5. A Counterknot? }
$$

Given the expected bipolar symmetry of the pulsar wind, as well as the symmetry suggested by the presence of a jet and a counterjet at X-ray wavelengths, the existence of a "counterknot" to the northwest of the pulsar would not be unexpected. No such counterknot is apparent in the data, however. To put a limit on the brightness of such a feature, we rotated the image about the pulsar, then added a fraction of the rotated image to the original. Figure $7 d$ shows that a counterknot with a brightness of $10 \%$ of the knot would be readily detected. Closer examination of the images indicates that a counterknot would not become undetectable until it dropped below about $5 \%$ of the strength of the knot.

A possible explanation for the lack of a counterknot is Doppler beaming of the radiation from the jet. The radiation emitted by particles in a relativistic flow is Doppler shifted and relativistically aberrated. This is the same effect used by Pelling et al. (1987) to explain the asymmetry in brightness between the front and back sides of the X-ray torus. If the particles are moving in a wind that has a speed $v_{w}$, and emitting isotropically in their rest frame, and the spectral index of the radiation is $\alpha\left(f_{v} \propto v^{-\alpha}\right)$, then the observed flux is related to the emitted flux by a factor of

$$
\frac{f_{\text {obs }}}{f_{\text {emit }}}=\left(\frac{\sqrt{1-\beta^{2}}}{1-\beta \cos \theta}\right)^{\alpha+2},
$$

where $\beta=v_{w} / c$ and $\theta$ is the angle between the direction of the wind and the line of sight, measured in the observer's frame.

To apply this effect to the knot and hypothetical counterknot, we begin with the assumption that these features would be associated with oppositely directed flows away from the pulsar. In this geometry, the knot is moving toward us at an angle of $\theta$ with respect to the line of sight, and any counterknot would be moving away from us at about the same angle. For such a case, the ratio of the brightness of the knot to the brightness of the counterknot would be amplified by Doppler beaming by a factor (e.g., Pearson \& Zensus 1987) of

$$
\frac{\left(f_{\text {knot }} / f_{\text {counterknot }}\right)_{\text {obs }}}{\left(f_{\text {knot }} / f_{\text {counterknot }}\right)_{\text {emit }}}=\left(\frac{1+\beta \cos \theta}{1-\beta \cos \theta}\right)^{\alpha+2} .
$$

Figure 11 shows the results of this calculation for the parameter space defined by $\theta, \beta$, and $\alpha$. Flow velocity is plotted against viewing angle. Solid contours show the relative amplification for $\alpha=1.1$ (no reddening). Dashed contours show the relative amplification for $\alpha=0.43$ (reddening from $\mathrm{Wu} 1981$ ).

The heavy solid contour and the crosshatched region in the upper left of Figure 11 show the region in the $\beta-\theta$ plane where relativistic beaming alone would be adequate to account for the upper limit on the brightness of a counterknot, assuming 


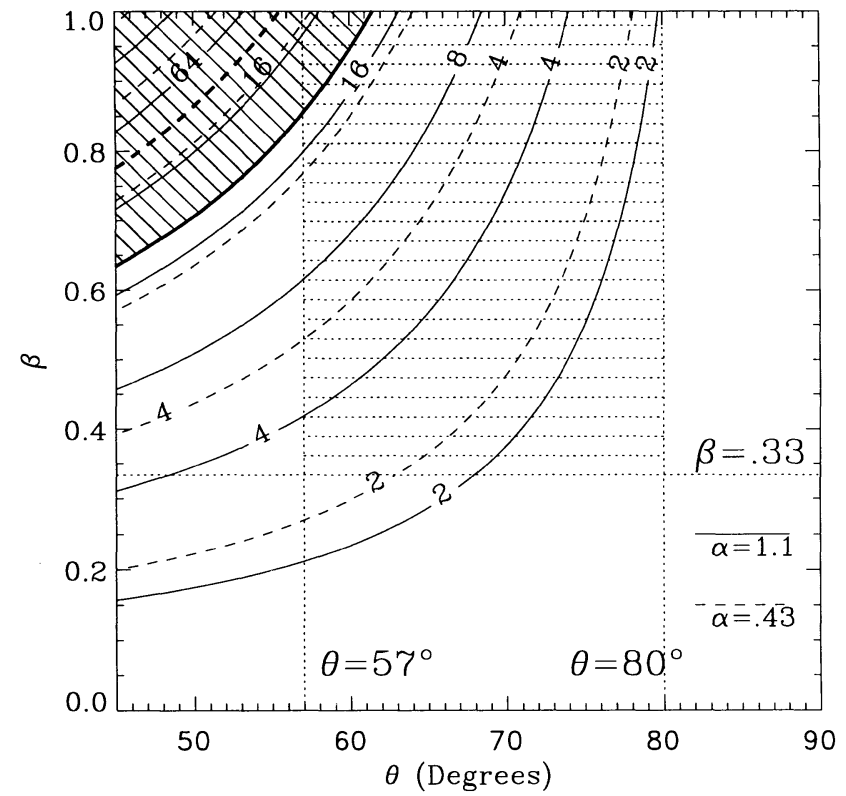

Fig. 11.-Contours show the effect of Doppler beaming on the ratio of the brightness of the observed knot and a hypothetical counterknot as a function of $\theta, \beta$, and $\alpha$. Flow velocity is plotted against viewing angle. Solid contours show the relative amplification for $\alpha=1.1$ (no reddening). The heavy solid contour and the hatched region at the upper left in the figure show where beaming alone could account for the observed upper limit on the brightness of a counterknot, assuming a spectral index of 1.1. Dashed contours show the relative amplification for $\alpha=0.43$ (reddening from $\mathrm{Wu} 1981$ ). The heavy dashed contour shows the observed upper limit on the brightness of a counterknot in this case. The lightly hatched region shows the likely range of values for $\theta$ and $\beta$. The dotted horizontal line is $\beta=\frac{1}{3}$. The vertical dotted lines mark limits of $\theta=57^{\circ}$ and $80^{\circ}$.

$\alpha=1.1$. The heavy dashed contour shows this limit for $\alpha=0.43$. The lightly hatched region shows the likely range of values for $\theta$ and $\beta$. The dotted horizontal line is $\beta=\frac{1}{3}$. The vertical dotted lines mark limits of $\theta=57^{\circ}$ and $80^{\circ}$. These come from the characteristics of the X-ray torus and the characteristics of the halo discussed in $\S 3.4 .8$ below.

As can be seen from the figure, there is very little overlap between the region of parameter space where beaming alone can explain the observed absence of a counterknot, and the range of parameters allowed by constraints on $\beta$ and $\theta$. A counterknot with an intrinsic brightness equal to the observed knot could exist only for $\beta \approx 1$, essentially no reddening, and an angle at the lower end of the range of estimates.

An important question, both for understanding whether a counterknot could be hidden by relativistic beaming and for understanding the nature of the knot itself, is the value of $\beta$ in the emitting material. The Lorentz factor of the pulsar wind must be $\gtrsim 10^{4}$ (see, e.g., Wilson \& Rees 1978), so the wind itself almost certainly has $\beta \approx 1$. If the mechanism responsible for the emission does not substantially slow the flow, then the asymmetry might be explained by beaming.

On the other hand, in many respects the knot can most easily be understood as downstream emission behind a strong shock in the polar wind. As discussed elsewhere, it is quite bright, covers a significant solid angle with respect to the pulsar, is very localized along the direction of the flow, and appears to persist with time $(\S 3.4 .4)$. For such a strong shock to exist at all may require that the flow be kinetic energydominated, in which case $\beta \approx \frac{1}{3}$ in the downstream flow (e.g.,
Kennel \& Coroniti 1984a). Assuming $\beta=\frac{1}{3}$, the beaming correction is only a factor of $\lesssim 3$, which would require that a hypothetical counterknot be intrinsically fainter than the observed knot by at least a factor of 7 .

\subsection{Temporal Variability of the Crab}

As discussed in $\S 3.3 .4$, the wisps appear to be well out of pressure equilibrium with their surroundings. Since the sound speed in this relativistic plasma is $\sim c / \sqrt{3}$, the sound crossing time for a wisp is $\sim 3.5 \times 10^{5} \mathrm{~s}$, or about 4 days. Such features must clearly be very dynamic.

Variability in the structure of the Crab synchrotron nebula has been known for some time. An especially nice discussion can be found in Scargle (1969), who examined a 13 yr sequence of Palomar plates dating back to 1955 . Prior to the discovery of the Crab pulsar, Scargle noted that the Crab in general and the wisps in particular were still " active." A program of temporal monitoring of the Crab using the WFPC2 could extend this study down to much smaller spatial and temporal scales. Such a study could be of particular importance in discriminating between changes in the structure of the Crab and true proper motion of features in the nebula.

\subsubsection{Comparison of WFPC2 and CFHT Images}

It is possible to begin a study of the evolution of the Crab at 0 .'5 resolution by comparison of the WFPC2 images with a very high quality ground based image of the Crab synchrotron nebula taken with the Canada-France-Hawaii Telescope (van den Bergh \& Pritchet 1989). Their data were taken on 1988 October 11, so the baseline between the CFHT and WFPC2 observations is $5.41 \mathrm{yr}$ or $1.70 \times 10^{8} \mathrm{~s}$.

To facilitate comparison between the two exposures we began by aligning the CFHT image with the WFPC2 mosaic using measured positions of 20 stars that fell within WF2. While this choice of alignment of stars results in a very slight misalignment in WF3, it gave very good alignment in the vicinity of the pulsar, and adequate alignment throughout the rest of the WFPC2 field of view to allow detailed comparison. Next, the resolution of the WFPC2 image was degraded to match that of the CFHT image by convolution with a 0.545 FWHM Gaussian kernel. Both frames were then masked so as to leave only the region within which both images contained good data, and a least-squares linear fit was computed between the two frames to determine the optimum scaling and offset to apply to the CFHT image to match it photometrically to the WFPC2 image.

The results of this are shown in Figure 12 (Plate 14). Figure $12 a$ shows the degraded WFPC2 image in the region of overlap between the two frames. Figure $12 b$ shows the CFHT image over the same field. Sharp structure in both the WFPC2 and the CFHT data has been emphasized by subtracting a smoothed version of their average from each. Figure $12 c$ shows the average of the two frames. Figure $12 d$ shows the difference between the CFHT and the smoothed WFPC2 images. A blowup of the region surrounding the pulsar is shown in Figure 13 (Plate 15).

\subsubsection{Temporal Evolution or Spectral Index Variations?}

Figure 12 shows significant differences between the WFPC2 and CFHT images. However, these differences cannot be unambiguously identified with temporal variations in the nebula without first addressing the question of differences in the appearance of the nebula between the $I$ and $V$ passbands 

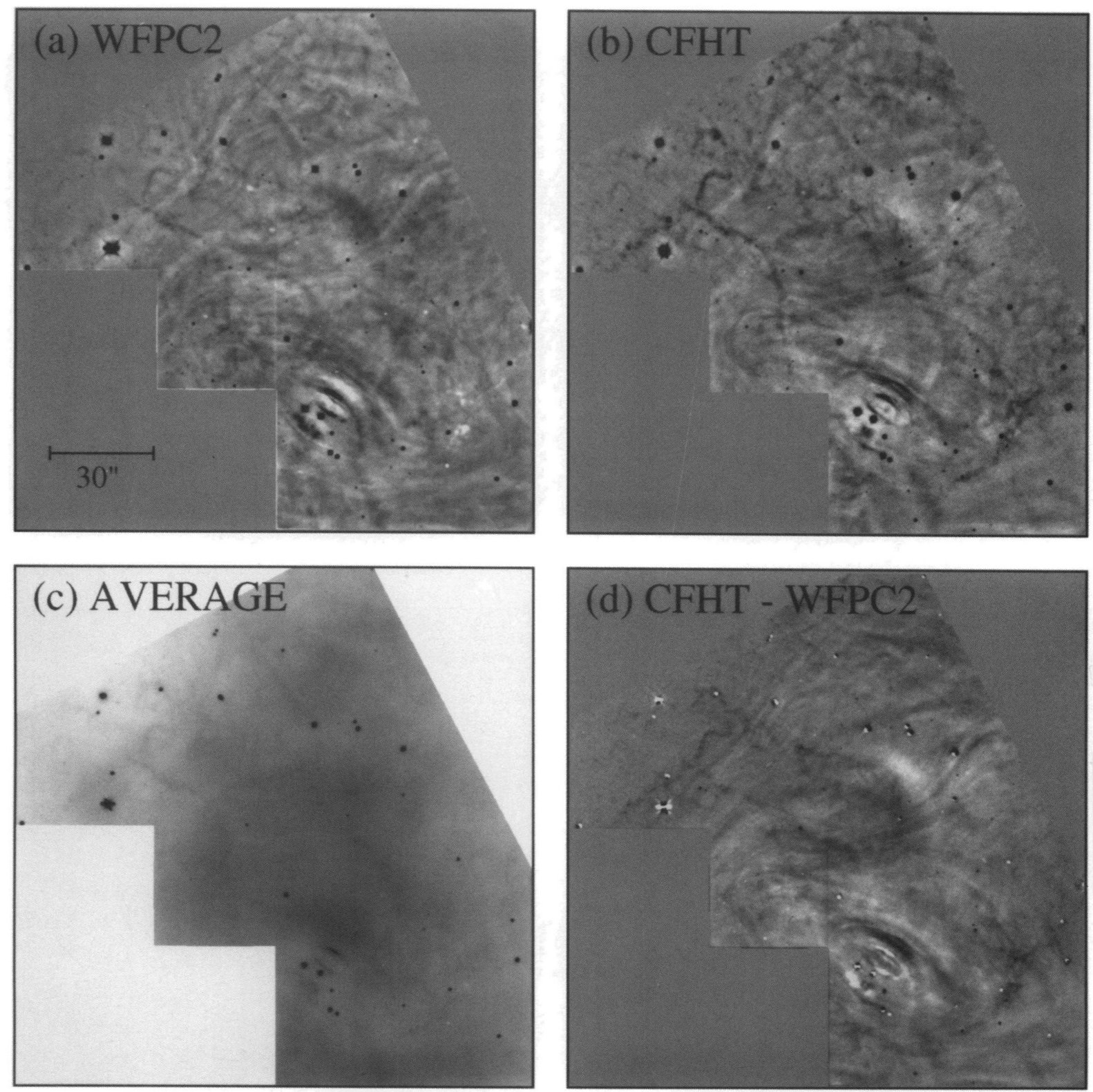

FIG. 12.-(a) WFPC2 image in the region of overlap with the CFHT image. The data have been smoothed to match the resolution of the ground-based data. (b) Portion of the CFHT I-band image published by van den Bergh \& Pritchet (1989) in the region of overlap with the WFPC2 field. In order to enhance comparison of small-scale structures in the two images, their average was smoothed with a 6" Gaussian and subtracted from each for display. (c) Average of the degraded WFPC2 and the CFHT images (prior to subtracting their smoothed average). (d) Difference between the two images. Regions where the CFHT image showed a higher signal than the smoothed WFPC2 image appear as dark.

HESTER et al. (see 448, 253) 

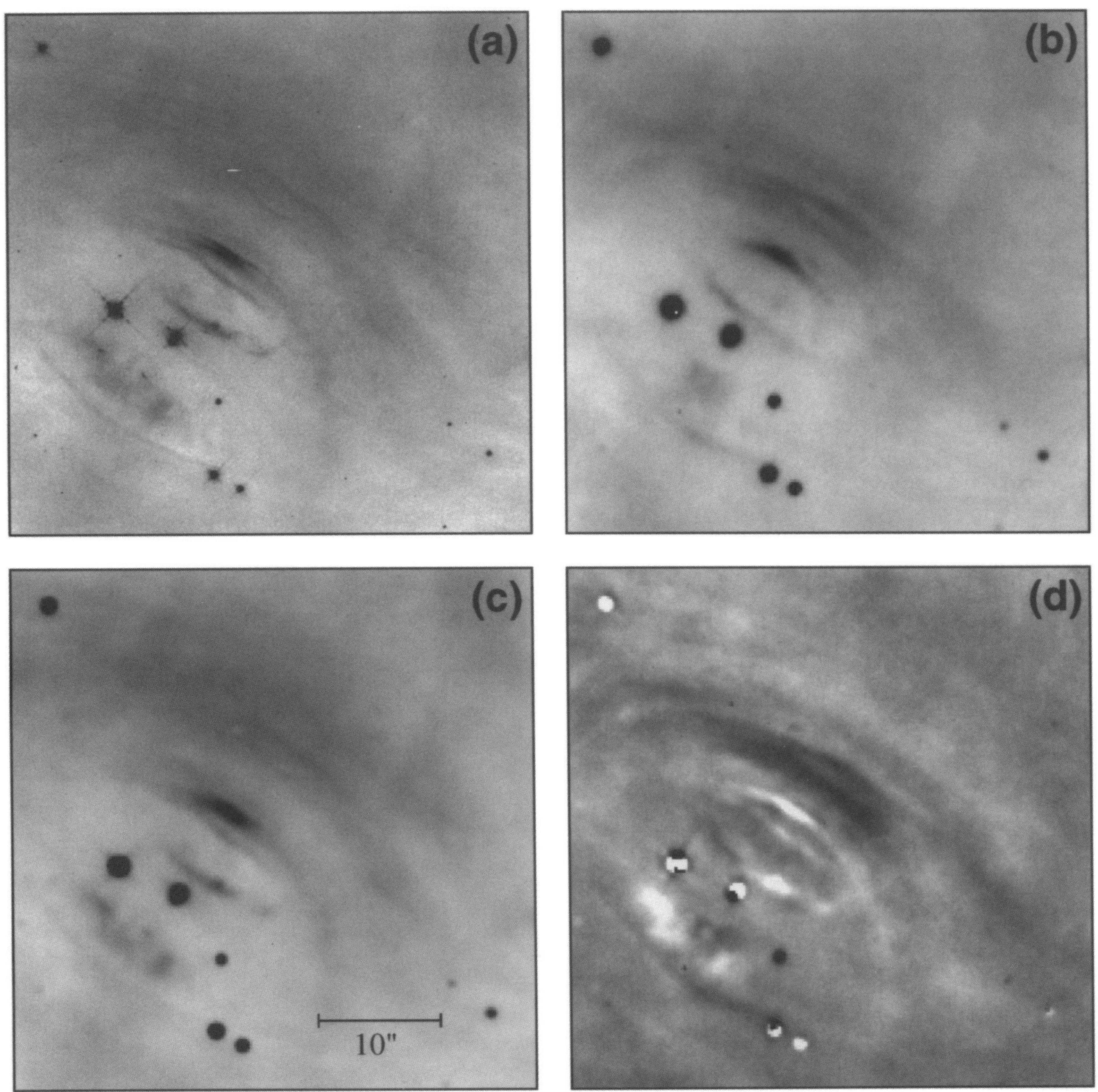

Fig. 13.-Comparison of high-resolution images of the Crab synchrotron nebula in the vicinity of the pulsar. (a) WFPC2 F547M image at full resolution. (b) The 0"5 resolution I-band image published by van den Bergh \& Pritchet (1989). (c) WFPC2 image, with resolution degraded to match the CFHT data. (d) Difference between the CFHT image and the degraded WFPC2 image. Dark areas are regions where the CFHT image was stronger.

HesTER et al. (see 448, 253) 
through which the images were taken. Previous studies of spectral index variations in the Crab in the visible and infrared (Hester et al. 1990) and radio (Bietenholz \& Kronberg 1992) have shown that spectral index variations do exist within the nebula on both large and small spatial scales. Fortunately, we can make a more direct test for possible spectral index variations by comparing the CFHT I-band data with the $5100 \AA$ PFUEI image presented above. These images were taken only $0.75 \mathrm{yr}$ apart. To make the comparison, we aligned the PFUEI image with the CFHT and WFPC2 images, then degraded the resolution in the CFHT and WFPC2 images to match the 2".5 resolution of the PFUEI data. The PFUEI image was scaled and offset to photometrically match the CFHT data. Differences were then taken among the three frames.

The results are shown in Figure 14. Figures $14 a, 14 b$, and $14 c$ show the low-resolution PFUEI, CFHT, and WFPC2 images, respectively. Figure $14 d$ shows the difference between the CFHT $I$ and PFUEI $5100 \AA$ images. Dark in this image corresponds to regions that were relatively stronger in the $I$ image than in the $5100 \AA$ image. Very little structure can be seen in these data. Some low spatial frequency structure exists, which roughly correlates with apparent spectral index variations seen in the $1.57 \mu \mathrm{m} / 5100 \AA$ ratio map presented by Hester et al. (1990). Most of the high spatial frequency features in the difference image are locations of emission-line filaments, which are faintly present in the $I$-band image but almost totally excluded in the $5100 \AA$ image.

The only significant high spatial frequency structure in the $I-5100 \AA$ image associated with the synchrotron nebula is very near the pulsar, where Scargle's wisps 1 and 2 are brighter in $I$ than in $5100 \AA$. It is not possible to say whether this difference is due to temporal changes in the structure of the nebula or to spectral index variations at small spatial scales, but it is likely to be a combination of the two effects. It is clear that the structure of the wisps can change significantly over 1 yr timescales, and so temporal variations cannot be ruled out. On the other hand, the IR/ $V$ ratio map presented by Hester et al. (1990) using data taken within a 5 day period also appears to show both of these wisps as stronger in the infrared.

For completeness, Figures $14 e$ and $14 f$ show the difference images of the PFUEI data minus the WFPC2 data and the CFHT data minus the WFPC2 data, respectively. These images are essentially identical, and both show a wealth of structure down to the 2 ".5 resolution of the data. On this basis, we conclude that, with the possible exception of differences in the strength (but probably not the position or basic morphology) of the inner wisps, the structure of the nebula at $I$ can be directly compared with the structure of the nebula at $5000 \AA$. Thus, the difference image in Figure 12 can be interpreted as temporal evolution of the nebula.

\subsubsection{Structure and Evolution of the Central Region}

As would be expected from previous studies (Scargle 1969), the most pronounced changes in the appearance of the Crab between 1988 and 1994 are in the central region (Fig. 13). The thin (1"8) wisp appears to have shifted position along its length. In the 1988 CFHT image, the northeast end of the thin wisp is more prominent, and there is a definite "hook" on this end. In the 1994 WFPC2 image, the northeast end of the thin wisp is no longer prominent. Rather, the central and southwestern portions of the wisp are more pronounced and show significant substructure. Note in particular the bright knot and sharp filament which have appeared to the west of the pulsar.
Also clear in the 1994 data is the fact that the southwest end of the faint wisp also turns away from the pulsar. As noted by van den Bergh \& Pritchet (1989), the data show the thin wisp to be clearly convex with respect to the pulsar, unlike the rest of the wisps. This is especially clear in the difference image. There has been no lateral shift (i.e., motion toward or away from the pulsar) in the position of the thin wisp between the two epochs.

Wisp 1 has remained the most prominent wisp, and the brightest portion of the wisp is in almost exactly the same position in the WFPC2 data as it was in 1988. This is a remarkable observation, because Scargle (1969) reported transverse velocities for wisp 1 of up to $0.3 c$. It seems unlikely that we have happened to catch wisp 1 at exactly the same phase in a periodic motion, and so we do not favor this interpretation. The details of the structure of wisp 1 have changed significantly, however. In 1988 wisp 1 appeared to be a single wisp, concave with respect to the pulsar, with a broadening through its brightest region. A second faint isolated filament was located a couple of arcseconds to the west of the brightest part of the wisp. In 1994 wisp 1 has split into three components (wisps $1 \mathrm{~A}, 1 \mathrm{~B}$, and $1 \mathrm{C}$ in $\S 3.3 .2$ above), the brighter two of which join in an elongated " $\mathrm{X}$ " at the location of the previous bright spot in wisp 1 . A more diffuse band of emission $\sim 2$ " thick has also appeared running along wisp 1 and extending on to the west and south of the pulsar. It is unclear whether this feature is truly an extension of wisp 1 or a separate structure.

The structure of wisp 2 has changed notably between the two images. In 1988 wisp 2 was a well-defined system of wisps with two especially strong components that joined together in a structure reminiscent of the appearance of wisp 1 in 1994. A third diffuse component was located about 2 " beyond the main two components. In the WFPC 2 images the features that made up wisp 2, including the fibers that make up the "X," appear to have shifted to the northwest by about 1 ". 8 . We cannot rule out the possibility that these are totally different features which have similar morphology due to their similar origin. If this is true proper motion, it corresponds to a velocity of $3200 \mathrm{~km} \mathrm{~s}^{-1}$ $(0.01 c)$ if it is in the plane of the sky, or $6400 \mathrm{~km} \mathrm{~s}^{-1}(0.02 c)$ if it is in the plane defined by the X-ray torus.

Aside from the possible shift in position of the features comprising wisp 2, these features are far less pronounced than they were in 1988. Rather than two or three especially prominent wisps, this region is now composed of a larger ensemble of very faint fibers embedded within a diffuse component (the " $\mathrm{T} 1$ wisps" discussed in $\S 3.3 .3$ above).

To the southeast of the pulsar, the counterwisp moved laterally very little between the two epochs, but, like the thin wisp, it has shifted along its length, becoming brighter toward its northeastern end and fainter toward its southwestern end. This type of behavior is not hard to understand. Assuming a restricted pitch-angle distribution of electrons, wisps will appear brightest where the magnetic field is perpendicular to our line of sight. In this case, ripples or shifts in the direction along which wisps point can cause some portions of a wisp to become more or less bright.

Some of the most pronounced changes in the structure of the emission near the pulsar occur in the vicinity of the anvil. In 1988 the anvil appeared to be a small trapezoid about $2^{\prime \prime}-3^{\prime \prime}$ on a side, much as it did in the images from which Scargle gave it its name. In the WFPC2 data, however, the anvil has become an amorphous region of emission approximately $10^{\prime \prime} \times 2$ ".5 in size. An unresolved knot has appeared toward the northeast end of the anvil. 

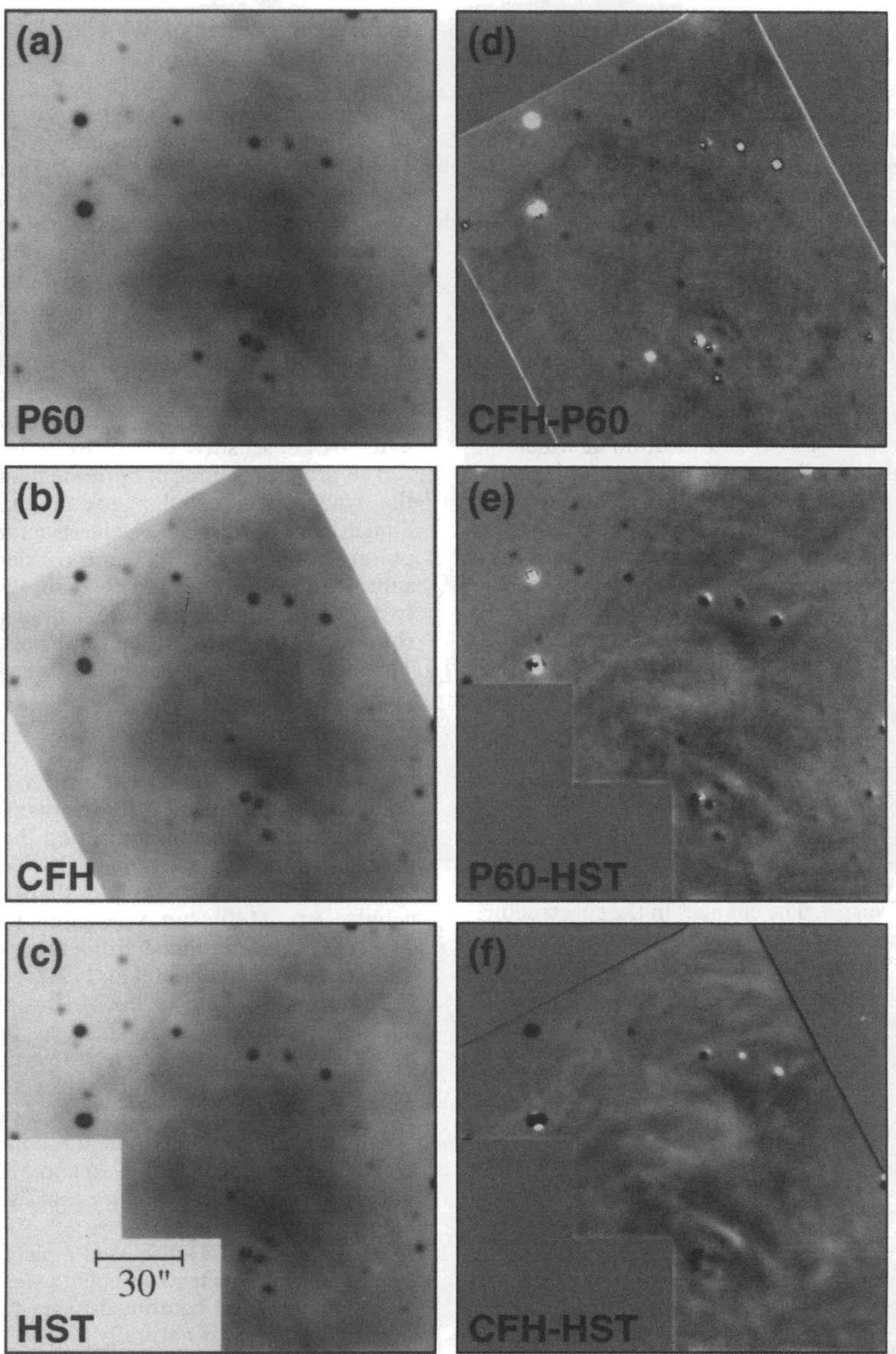

Fig. 14. - Comparison between WFPC2 V, CFHT I, and Palomar 60 inch $(1.5 \mathrm{~m})$ PFUEI images of the Crab. (a) The $5100 \AA$ Palomar 60 inch image of the Crab (epoch 1988.03). (b) Van den Bergh \& Pritchet's (1989) CFHT I-band image of the Crab (epoch 1988.78), with resolution degraded to match the 2".5 resolution of the PFUEI image. (c) WFPC2 F547M image of the Crab, with resolution likewise degraded. (d) Difference between the CFHT and the Palomar 60 inch images. Features which are strong in the $I$-band image appear as dark in the image. These two frames, taken within $0.75 \mathrm{yr}$ of each other, show few differences, despite the difference in passband. (Exceptions include residual line emission from filament emission in the $I$-band data, slight low spatial frequency spectral index variations, and an apparent difference in the strength of the inner two wisps. See text.) In $(e)$ and $(f)$ are shown the PFUEI - WFPC2 and CFHT - WFPC2 difference frames, respectively. The lack of structure in $(d)$ and the similarity of the structure in $(e)$ and $(f)$ show, with the possible exception of the strength of the inner wisps, that the changes seen at higher resolution between the WFPC2 and CFHT images are predominantly due to temporal evolution of the nebula.

\subsubsection{Changes in the Knots?}

It would be very interesting to know the temporal evolution of the two knots discovered in the WFPC2 image. While neither of these knots can be seen clearly in the CFHT data, both in fact appear to be present. There is an unresolved condensation in the anvil in the CFHT data located within about
0.2 of the location of the outer WFPC 2 knot. In addition, under visual inspection the pulsar image appears to be slightly elongated in the CFHT image in the direction of the inner knot. To test this, profiles were taken across the pulsar image and across three unsaturated star images located close to the pulsar. The profiles of the star images were all symmetrical, 
with measured FWHM widths of 0 ".58 \pm 0 ".01. The pulsar image, however, shows a clear tail to the southeast, and has a measured single-component width of 0 "'64. When fitted with two Gaussian components, the fitting algorithm places the second component a distance of 0 ".66 from the primary component, in good agreement with the separation between the pulsar and the knot measured from the WFPC2 image.

Thus, we tentatively conclude that both of the knots were present in 1988, and have changed position very little since then. (Once again, we cannot rule out the possibility that the inner knot has formed and dissipated or formed and moved outward many times since 1988 , and that by chance we have caught this process in the same phase as before.) Assuming that the knots are due to disturbances in the pulsar jet, their apparent stability suggests that they are the result either of welldefined wave modes or of a well-defined location at which the flow develops an instability. This conclusion awaits repeated observations with the HST for confirmation. However, if it holds true, it is good news from the standpoint of possible theoretical interpretations because it means that the knots and their location are a stable property of the polar jet.

\subsubsection{Change versus Motion}

As noted in the caveats above, it is difficult to know whether apparent shifts in position of features in the inner synchrotron nebula are true proper motions or whether they are due to changes in the structure of the nebula (e.g., phase shifts in some wave phenomenon in the pulsar wind).

Scargle (1969) spent considerable effort discussing the difference between change and motion when interpreting observations of the inner Crab. In the end he generally favored "motion," largely on the basis of the possibly periodic shifting about of the position of wisp 1 . The changes in the substructure of wisp 1 seen here, however, seem to favor the alternative interpretation. Wisp 1 shows complex and rapidly evolving substructure which could appear to be a single moving feature when viewed at low resolution.

We are forced to conclude that while we are able to show that the variations in appearance noted by Scargle are even more fascinating at higher spatial resolution, the current study at 0.5 resolution covering a 5.41 yr baseline may still be based on two random and (in the case of the WFPC2 image, intentionally) blurry snapshots of sharper structures that are changing on much shorter timescales. Definitive statements about the evolution of the Crab await data with 0 ". 1 resolution and temporal sampling of timescales down to a week or shorter.

\subsubsection{Changes in the Outer Nebula}

Changes in the outer nebula are generally not as dramatic as those in the inner nebula, but many are still apparent. The most pronounced of these is a large region located about $30^{\prime \prime}-1^{\prime}$ north of the pulsar (Fig. 12d). This is not a spectral index feature (see Fig. 14d). There is a horseshoe-shaped region (open to the northeast),which was bright in 1988 (Fig. 12b) but dark in 1994 (Fig. 12a). Immediately to the north of this horseshoe is a broad arc, which was faint in 1988 but bright in 1994. Again, the nature of this feature or the way in which it evolves is unclear.

Things are less grim elsewhere. There are a number of largescale striations in the outer nebula that have clearly maintained their identity but changed their position between the two epochs. We selected one such feature somewhat to the south of the center of WF4 in the WFPC2 data to use to measure the proper motion of material within the synchrotron nebula. This feature can be traced almost across the entire diagonal of WF4 (see Fig. 9). It was chosen in part because it passes $\sim 8^{\prime \prime}$ behind the prominent hook-shaped filament of ejecta to the north of the pulsar. This allowed us to measure both the proper motion of ejecta and the proper motion of the synchrotron structure at about the same projected distance from the pulsar. (These two features are apparent in Fig. 12d.) This feature is also roughly perpendicular to the direction of the pulsar, which removed much of the ambiguity about motions along the striation. Finally, we note that the line from the pulsar to these features is roughly perpendicular to the direction of the pulsar's proper motion (Wyckoff \& Murray 1977), so determinations of radial distances and expansion dates are not sensitive to choice of expansion center.

The proper motions of both the filament and the striation in the synchrotron nebula were measured by extracting onedimensional profiles across the structures (pointing back at the pulsar) in both the CFHT and the degraded WFPC2 images, subtracting off a linear fit to each, then cross correlating the two profiles. (Similar measurement of star images in the region showed misalignment along this direction of only about 0 ".01.) The filament of ejecta was located at a distance of $84^{\prime \prime}$ from the pulsar in 1988, and had an observed proper motion of 0".59 over the $5.41 \mathrm{yr}$ baseline between the two images. This corresponds to a velocity of $1000 \mathrm{~km} \mathrm{~s}^{-1}$ and an expansion time $(r / \delta r) \delta t$ of $770 \mathrm{yr}$ (dated back from 1988). This gives a convergence date of A.D. 1218 for the filament, $164 \mathrm{yr}$ after the date of the actual explosion, indicating that the filament has been accelerated as originally noted by Trimble (1968). Our convergence date is somewhat later than Trimble's average for the nebula (A.D. 1140), but very close to the convergence date ( A.D. 1245) determined from the overall expansion of the radio nebula (Bietenholz et al. 1991).

The proper motion of the synchrotron feature gives a very different result, however. This feature, located $78^{\prime \prime}$ from the pulsar in 1988, experienced a proper motion of 1".01. This corresponds to a velocity of $1720 \mathrm{~km} \mathrm{~s}^{-1}$ in the plane of the sky, an expansion time of $407 \mathrm{yr}$, and a convergence date of A.D. 1581 , over $330 \mathrm{yr}$ later than the convergence date from the synchrotron nebula as a whole and $527 \mathrm{yr}$ after the explosion. This feature is clearly moving rapidly through the interior of the synchrotron nebula.

Michel et al. (1991) discussed a picture of the Crab in which the expanding synchrotron nebula was "caged in" by the filaments of ejecta but continued to try to "pooch through" this cage. (This picture is naturally able to account for the fact that the synchrotron nebula is slightly larger than, but still in about the same place as, the filaments - the observation that led Bietenholz et al. 1991 to what we believe may be an erroneous conclusion that the synchrotron nebula is currently bursting through the filaments into the surrounding ISM.) This picture was used to explain such features as the coincidence of the western bay in the synchrotron nebula and a bright ejecta filament at this location. This view was developed by Fesen et al. (1992), who suggested that the western and eastern bays were tied together by a presupernova disklike ejection which is also responsible for the "high-He" band discussed by Uomoto \& MacAlpine (1987).

In the picture discussed by Michel et al., the especially strong polarization seen along the bays and elsewhere in the nebula was due to the magnetic field within the nebula piling 
up at the filaments. The new observations fit this model very well. Rather than a homologously expanding structure, the interior of the Crab Nebula is a region of energy transport between the pulsar and the filamentary cage. Flux tubes move rapidly through the nebula, piling up on the surface of the filamentary cage, where they deposit their energy. These structures then "cool," both by radiating and by doing PV work on the filaments. (The Rayleigh-Taylor unstable interface at the edge of the Crab as revealed by WFPC2 observations of the filaments has been discussed by Hester et al. 1994. This, as well as the effect that the work done on the filaments by the synchrotron nebula has on the energy budget of the nebula, will be discussed in a subsequent paper.)

\subsubsection{The Nature of Structure in the Outer Nebula}

Following the arguments in $\S 3.3 .4$, we find equipartition fields for the low-contrast features in the outer nebula to be about a factor of 2 higher than the canonical value for the Crab. This implies that the equipartition pressure is a factor of 4 higher than the average equipartition pressure for the nebula as a whole.

It is not surprising that the bright wisps near the pulsar are out of equilibrium with the rest of the nebula. As originally suggested by Rees \& Gunn (1974), the wisps almost certainly arise where the flow velocity of the pulsar wind is thermalized (i.e., at the wind shock), and particles accelerated there probably move rapidly along field lines out into the nebula. The existence of persistent structure in the outer nebula is more troublesome. Given the high sound speed in the plasma, pressure deviations with the observed size scales should be washed out in a matter of weeks.

If the structures in the outer nebula are not due to pressure differences, then they must instead be due to differences in the volume emissivity of the plasma; differences in volume emissivity at constant pressure would in turn imply departures from equipartition. Since the synchrotron volume emissivity of the plasma has a maximum at equipartition, the equipartition pressure derived above is a lower limit on the true pressure of the nebula. In the region where this structure arises, the true pressure of the nebula is at least a factor of 4 higher than the equipartition pressure calculated from the average volume emissivity of the nebula as a whole.

In this view, bright features in the nebula represent regions which may be close to equipartition, while faint features represent regions which are not. Since particles are free to move along the magnetic field, the degree of deviation from equipartition (and hence the volume emissivity) will be roughly constant along field lines, confirming our assumption that the fibrous texture of the nebula traces the coherent large-scale structure of the magnetic field.

We speculate that these outer features may be the remnants of "flux tubes" formed by instabilities at the wind shock that have expanded and coalesced as they advected out through the nebula. The origin and evolution of these magnetic structures are an interesting theoretical problem.

\subsubsection{A Halo?}

When the CFHT and WFPC2 images of the thin wisp and wisp 1 are compared, they appear to close into a halo-like structure above the pulsar. Note in particular the appearance of the thin wisp, which curves toward wisp 1 on the northeast end in the older data and on the southwest end in the newer data, and the existence of an arc connecting the southwest end of the two wisps in the WFPC2 data. Certain identification of this halo as a physical structure awaits more epochs of high resolution data. At the moment, for example, we cannot rule out the possibility that the "closure" at the two ends is simply due to a cusp where the two wisps cross each other in projection. On the other hand, the emission on both ends seems to curve back on itself. Further, the physical characteristics of the thin wisp and wisp 1 are very similar. Both have about the same emissivity (see Table 1), and these two wisps also have the most stable locations of any features in the vicinity of the pulsar. We therefore think it likely that these features are part of a single physical structure.

Note that we do not propose that wisp 2 or the other structures running through this region are part of the halo. Rather, we believe that these structures are generally part of the larger torus, as discussed above. Our suggestion requires that the near side of the halo (wisp 1) be viewed in projection near the southeastern edge of the near side of the torus. Similarly, it is tempting to associate the counterwisp with a halo to the southeast of the pulsar, but on the basis of the radius of curvature and general appearance of this feature, we believe that it is likely on the back side of the torus.

If we interpret wisp 1 and the thin wisp as the front and back portions of a halo, we can infer some of the physical characteristics of the halo. The dimensions of the halo are approximately $13^{\prime \prime} .7 \times 5$." 0 , although these numbers, especially the long dimension of the halo, are uncertain. Assuming that the halo is actually circular, the radius of the halo is $\sim 2.1 \times 10^{17}$ $\mathrm{cm}$, and its elongation implies that the axis of the halo is tipped by $21^{\circ}$ with respect to the plane of the sky. Given the uncertainties, this is close to the $33^{\circ}$ tilt inferred for the X-ray torus. In addition, the axis of the halo is fairly well aligned with the visible knots, the axis of the X-ray torus, and the X-ray jet and counterjet. If we assume that the axes of the X-ray torus and the visible halo are close to parallel, then the wisp 1 side of the halo is toward us and the thin wisp side of the halo is the back side. We presumably see wisp 1 and the thin wisp rather than the entire halo because of synchrotron beaming.

The center of the halo is located $~ 4$ ". 4 from the pulsar, or a distance of $1.3 \times 10^{17} \mathrm{~cm}$ on the plane of the sky. Assuming that the axis is tilted by $21^{\circ}$ then gives a physical distance of $1.4 \times 10^{17} \mathrm{~cm}$ between the pulsar and the plane of the halo. This, combined with the size of the halo, implies that the opening angle of the cone defined by the pulsar and the halo is $110^{\circ}$. The halo then appears to be a stable structure located at a latitude of $35^{\circ}$ with respect to the pulsar.

Based on the lengths of the wisps, we estimate that the pitchangle distribution of electrons in the halo is concentrated in a range of $\sim \pm 30^{\circ}$. The ratio of the lengths of wisp 1 and the thin wisp to the length of the optically prominent portion of the torus to the northwest of the pulsar is about equal to the ratio of the inferred radii of the halo and the torus. Thus the pitchangle distributions of particles in these two regions would seem to be similar. Obversely, this supports our interpretation of the halo as a physical structure. If wisp 1 and the thin wisp were part of the torus, it would imply a much narrower $\left( \pm 7^{\circ}\right)$ pitchangle distribution in these features than elsewhere in the torus.

As discussed in $\S 3.3 .4$, the energy density in the features that make up the halo is very high. By analogy with laboratory experiments that show the difficulty in magnetically confining plasmas, it might be expected that instabilities in the halo would open channels through which particles could escape into the nebula. One such "breakout channel" may be visible in our data. This feature (marked in Fig. 9) is an irregular $\sim 1^{\prime \prime}$ 
thick feature that emerges from wisp 1, then takes a sharp turn $\sim 10^{\prime \prime}$ south of the pulsar and extends radially away from the center of the nebula. This feature can also be seen in the ground based data (Fig. $3 d$ ) and the ROSAT image (Fig. $3 b$ ) as a sharp elongated feature just to the southwest of the pulsar. The structure appears to join continuously with the X-ray feature OS. In $\S 3.1 .3$ OS was interpreted on the basis of its association with an outward dimple in the magnetic structure of the nebula as a channel through which energy was flowing through the nebula. Here we speculate that we may have traced that energy flow back to its source in the wind shock.

\subsubsection{A Channel around the Counterjet}

The visible structure of the nebula may support the interpretation that the X-ray jet and counterjet are true jets, i.e., directed flows of energy. We have already mentioned a cap of $\mathrm{X}$-ray emission which appears to be material compressed by the counterjet. The comparison between the CFHT and WFPC2 data also shows that the visible structure which runs parallel to the counterjet has a proper motion away from the counterjet rather than away from the pulsar. The data suggest that the counterjet is forcing material out of its way.

Near the base of the counterjet there are also two sets of features that parallel the counterjet on either side. These features can be seen clearly in the high-pass filtered WFPC2 and CFHT data (Figs. 2, 12a, and 12b) running southeast to northwest across the grain of the wisp 2 region. Each feature is $\sim 10^{\prime \prime}$ long and consists of two to three alternating bands, each $\sim 2^{\prime \prime}$ across. These features are located approximately $10^{\prime \prime}$ on either side of the axis of the counterjet, and roughly bound the channel of low polarization to the northwest of the pulsar reported by Hickson \& van den Bergh (1990). The features also project back to just outside the northeastern and southwestern tips of the visible halo discussed in the previous section.

Taken together, the data (especially the features near the base of the counterjet) seem to suggest the existence of a channel $\sim 20^{\prime \prime}\left(6 \times 10^{17} \mathrm{~cm}\right)$ in diameter encasing the X-ray counterjet.

\section{THE PULSAR/NEBULAR CONNECTION}

\subsection{The Cylindrical Symmetry of the Crab}

We begin our discussion of the relationship between the Crab pulsar and the synchrotron nebula by trying to build a basic observational picture of the environment surrounding the pulsar. We find that the most fundamental observation to be made about the structure of the Crab Nebula is that almost all observations of the system at all scales show a well-defined axis of cylindrical symmetry running from the southeast to the northwest through the center of the nebula, at an angle tilted by $20^{\circ}-30^{\circ}$ with respect to the plane of the sky.

This suggestion is not new. The existence of this axis has been noted by a number of authors in a variety of contexts. This axis corresponds to the direction of elongation of the nebula as a whole, the axis of the X-ray and optical jets and $X$-ray counterjet, the $X$-ray torus, and the alignment of the wisps. Kronberg et al. (1993) have found that the spatial variations in radio spectral index in the Crab can be modeled by circular structures about this same axis. Begelman \& Li (1992) constructed MHD models which have this symmetry. Most recently, Fabry-Perot data presented by Lawrence et al. (1994) beautifully show that even the filamentary ejecta are concentrated in a cylindrical band which lies along this same axis.

In the present paper we report a number of additional phe- nomena, including the knots $(\S 3.3 .1)$, the halo $(\S 3.4 .8)$, the channel surrounding the counterjet $(\S 3.4 .9)$, and the fine texture of the nebula $(\S 3.3 .3)$, all of which share this same symmetry. The nebula is powered by the spin-down energy of the pulsar, and the only physical axis that exists for the pulsar is its spin axis. It seems almost certain that the structure of the region immediately surrounding the pulsar must reflect this symmetry (e.g., Aschenbach \& Brinkmann 1975). By strengthening the observational link between the symmetry of the pulsar's immediate surroundings and that of the nebula overall, we have better established the tie between the spin axis of the pulsar (and/or the progenitor) and the structure of virtually all observed aspects of the Crab.

The only significant published counterexample to this symmetry is the suggestion of a north-south axis through a prominent ring of ejecta filaments running east to west across the nebula (Uomoto \& MacAlpine 1987). This ring was recently discussed at length by Fesen et al. (1992) in connection with the origin of the synchrotron bays. This north-south symmetry also appears in an hourglass-shaped region of high polarization noted by Michel et al. (1991).

This east-west ring may or may not be of fundamental significance to the overall structure of the nebula. Data presented by Lawrence et al. (1994) show the existence of other "rings" of filaments which would come into prominence if viewed from different angles. We suggest that the east-west ring may have attracted attention primarily because it happens to be aligned along our line of sight. This fortuitous alignment would also explain the prominence of the east and west bays, since only in these locations would the scalloping of the synchrotron nebula by its interaction with the filaments (Michel et al. 1991) lie along our line of sight. Li \& Begelman (1992) have suggested that the hourglass polarization structure may result from distortions of the magnetic field structure by this ring of filaments. This is a plausible explanation that would apply regardless of whether these filaments are associated with a second symmetry axis, a wind from the precursor, or simply the chance alignment of the filaments with our particular perspective.

\subsection{An Observational Picture of the Inner Crab}

Having added to the evidence linking the overall structure of the Crab to the spin axis of the pulsar, we suggest that the inner synchrotron nebula must reflect latitudinal structure in the wind from the pulsar. We begin by enumerating the observed latitudinal zones and their properties. The existence of these zones then provides clear constraints on models of the wind from the Crab pulsar and its interaction with the synchrotron nebula.

Observationally, there are three wind zones:

1. The equatorial zone extends $\sim \pm 10^{\circ}$ from the spin equator of the system. The equatorial wind shocks at a distance of $\sim 1.2 \times 10^{18} \mathrm{~cm}$ from the pulsar. This zone is identified with the X-ray and visible torus, networks of very sharp visible fibers or striations, and much of the spectral index structure seen at radio wavelengths. Based on the emissivities of the visible fibers, the postshock pressure in this zone is $\sim 10$ times the nebular average. This zone shows significant substructure, including two distinct "branches" seen in both X-ray and visible data.

2. A high-latitude wind zone is found at latitudes greater than $35^{\circ}$. To the southeast of the pulsar this high-latitude wind shocks at a $z$-distance of $1.4 \times 10^{17} \mathrm{~cm}$ from the pulsar, giving 
rise to the anvil. The appearance of this region is extremely variable, although the distance between the pulsar and the anvil seems to be relatively stable. The X-ray/optical jet and the X-ray counterjet appear to originate at least in part in the wind shock in this zone.

To the northwest of the pulsar there also appears to be a high-latitude wind zone covering the same latitudes, but its morphology is completely different from that of the zone to the southeast. While there is a small amount of "anvil-like" emission in this region, it is much less pronounced than the amorphous emission to the southeast. This zone may be surrounded by a halo of wisps that lie near the boundary between the equatorial and high-latitude wind zones. The plane of the proposed halo is also $1.4 \times 10^{17} \mathrm{~cm}$ above the pulsar, similar to the anvil. There are a number of features that appear to be extending out of the northwest high-latitude wind zone in a direction parallel to the rotation axis. These may mark the sides of a channel formed by the X-ray counterjet.

3. There is apparently a more collimated polar wind zone, or jet, embedded within the high-latitude wind zone. To the southeast, the polar jet must shock at a distance of $\sim 1500 \mathrm{AU}$ $\left(2.3 \times 10^{16} \mathrm{~cm}\right)$ from the pulsar to account for the knot and arc seen there. To the northwest, however, there is no such knot. The second sharp knot, located $1.36 \times 10^{17} \mathrm{~cm}$ to the southeast of the pulsar, indicates that the polar jet probably remains well collimated to this distance.

In addition to the existence of these wind zones, models are constrained by the various determinations of emissivity, field strength, pressure, thickness of structures, proper motions (or at least variability) of features, polarizations, and spectral index variations discussed here and elsewhere. The northwest/ southeast asymmetry of the pulsar environment is also very pronounced and probably demands some asymmetry in the source itself.

\subsection{Digression: The Structure of Pulsar Winds}

Any discussion of the structure of pulsar winds should be prefaced with the statement that, despite considerable study, very little is really known about the nature of what comes off of a pulsar, apart from the fact that it must carry the energy and angular momentum lost by the pulsar as it spins down.

The two limiting cases for pulsar winds have been discussed at length in the literature. In an aligned rotator (Goldreich \& Julian 1969) the pulsar has a toroidally magnetized wind that is advected away. The sense of the polarization of the field changes between the two hemispheres, requiring polar and equatorial currents to flow. This solution also allows for the formation of helically polarized polar jets that carry energy, angular momentum, and electric current (Sulkanen \& Lovelace 1990). In the case of the oblique rotator (Gunn \& Ostriker 1971) the wind is in the form of azimuthally polarized waves.

The more realistic case of a partially aligned rotator is a more difficult problem. This case was treated approximately by Michel (1971), who showed that the result is a superposition of the two extremes, with an equatorial "striped" magnetic wind. This wind solution has been discussed more recently by Coroniti (1990) and Michel (1994), who primarily consider the problem of how a wind that is expected to emerge from the pulsar dominated by the energy in the field (e.g., Michel 1982) converts itself into the particle-dominated wind required by MHD models of the interaction between the wind and the nebula. This problem is generally characterized in terms of a parameter $\sigma$, defined to be the ratio of the Poynting flux to the particle kinetic energy flux in the wind upstream of the shock. Various treatments (Rees \& Gunn 1974; Kennel \& Coroniti 1984a) find a value of $\sigma \approx 10^{-3}$. Coroniti (1990) found that field reconnection in the striped magnetic wind would effectively convert field energy into particle thermal energy. Michel (1994) recovered this same result, but with a more intuitive approach, showing that the source of "reconnection" in this case was simply inductive heating of the particles.

A detailed discussion of the structure of the pulsar wind is well beyond the scope of this paper. However, to facilitate possible identification of observed features in the Crab with different regions in the wind, we include two figures which capture the essential qualitative features of the wind from an oblique rotator. Figures 15 and 16 respectively show pole-on and equatorial views of the fields and currents surrounding the pulsar. The essential features of the wind are as follows.

The DC component of the magnetic field is split into two oppositely polarized hemispheres, as in the Goldreich \& Julian (1969) model. This requires that a current flow in (out) the poles and out (in) the equator if the dot product of the magnetic dipole and the angular momentum of the neutron star is positive (negative). These currents must close either in the wind or in the nebula. In addition to polar currents, there is a strong Poynting flux directed away from the poles of the neutron star, as can be seen by considering the pole-on view of the system. The polar wind will also have a component of the helical jet discussed by Sulkanen \& Lovelace (1990).

At high latitudes the DC component of the magnetic field dominates the wave component, giving rise to a helical wind zone. Finally, near the equatorial plane is the zone where the striped magnetic wind solution applies. This is a zone where superposed on the DC component of the magnetic field is a strong wave component, in which stripes of alternating magnetic polarity are separated by current sheets. It is these current sheets that grow at the expense of field energy, converting the wind near the pulsar from a high- $\sigma$ wind to the low- $\sigma$ wind required to provide the observed shock at the interface between the wind and the nebula.

\subsection{Identification of Features with Wind Zones}

The basic understanding of pulsar winds outlined in the previous section leads to a picture that is azimuthally symmetrical but which has very different properties as a function of pulsar rotational latitude. This same description-azimuthally symmetrical but with significant latitudinal structure - is also a good summary of the observational picture of the Crab which we have presented. An obvious next step is to attempt to match the observed latitudinal features of the Crab pulsar's wind with those found in the theoretical treatments.

Figure 17 ascribes a plausible relationship between the observed features near the center of the Crab and the features in the wind model. As previously suggested by Coroniti (1990), the $\mathrm{X}$-ray torus is identified with the shocked equatorial wind. However, rather than occurring at a distance of $\sim 10^{\prime \prime}=3 \times 10^{17} \mathrm{~cm}$ from the pulsar (i.e., the projected distance to wisp 1), as is often assumed, the wind shock is instead located at a distance of $\sim 1.2 \times 10^{18} \mathrm{~cm}$, consistent with the semimajor axis of the torus.

The structure of the torus is complex at both X-ray and optical wavelengths. The torus seems to split into two components along its front face. The relatively fainter region between these two components may be identified with the equatorial plane of the system. This plane separates the two 


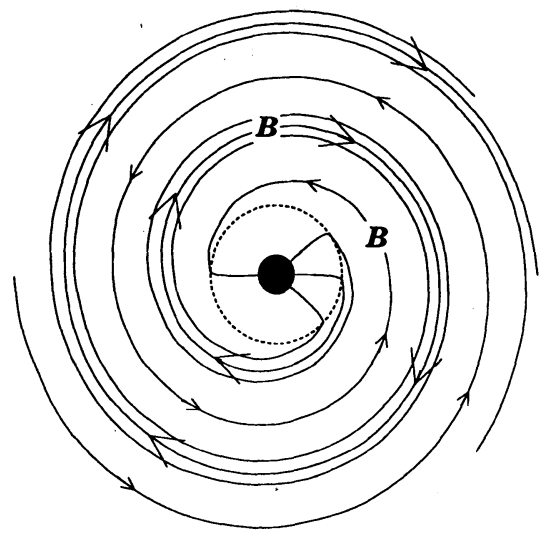

Geometry of the Magnetic Field

The view shows the field geometry

in one hemisphere only.

The sign of the DC field and polar current is reversed in the opposite hemisphere.

The sign of the AC fields and currents are the same in the opposite hemisphere.

The equatorial region is dominated by Coroniti's striped magnetic wind. The polar region is dominated by the Poynting flux from the rotating dipole.

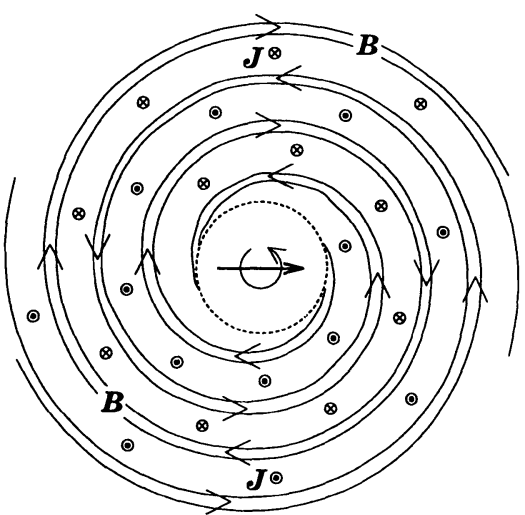

The AC component of the B field and currents. Along the axis the circularly polarized Poynting flux from the rotating dipole dominates.

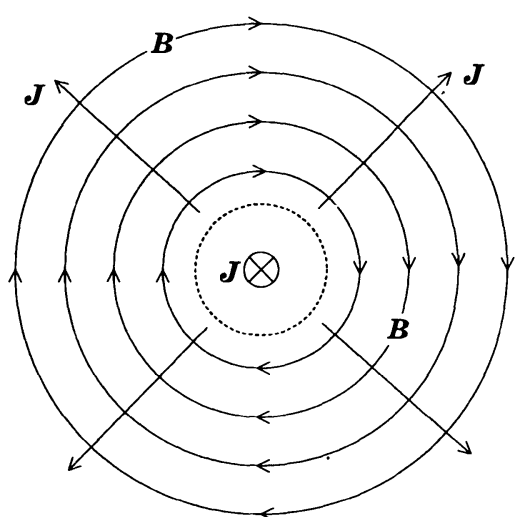

The DC component of the B field and current. $B$ is azimuthal. $J$ is in along the poles and out in the equatorial plane.

FIG. 15.-Polar view of the magnetic field geometry of the wind from an oblique rotator

hemispheres with oppositely polarized helical DC fields, and so the net field at this location should be a minimum. Coroniti notes that the rotator cannot be totally oblique in the Crab, because if it were, then the $\sigma$ of the wind would vanish. However, just such a zone where the average magnetic field is zero must exist around the equatorial plane of the wind.

The knot 0.7 to the southeast of the pulsar presumably is due to either a shock or some instability in the high-latitude wind from the pulsar. While this can possibly be identified in part with the helical wind, the fact that the outer knot is about the same size as the inner knot indicates that at least out to a distance of $1.4 \times 10^{17} \mathrm{~cm}(9100 \mathrm{AU})$ the jet has a more sharply defined core. The absence of a similar knot to the northwest of the pulsar $(\S 3.3 .5)$ breaks the symmetry in the flow from the two poles of the Crab pulsar - a feature not found in pulsar models. We suggest that one way to introduce such an asymmetry would be to have the magnetic axis of the pulsar not only misaligned with respect to the spin axis but offset from the center of the pulsar as well.

Interpretation of the possible halo and of the emission associated with the anvil is more troublesome. This is in part due to the notable asymmetry between the high-latitude emission to the northwest and southeast of the pulsar. However, we note that if the cone drawn through the pulsar and the halo is extended through the pulsar to the other side, it encircles the current extent of the anvil emission nicely. Thus, while there is clearly an asymmetry between the northwest and southeast regions near the jet, it seems likely that both the halo and the anvil are due to the same wind zone. This is possibly an important observation from the standpoint of constraining wind models, because it defines a transition about a rotational latitude of about $35^{\circ}$, above which the character of the wind shock changes. Further, the anvil, the halo, and the outer knot-i.e., all of the high-latitude features observed, with the exception of the inner knot-are located at very nearly the same distance $\left(\sim 1.4 \times 10^{17} \mathrm{~cm}\right)$ from the pulsar.

The arc within which knot 1 is embedded appears to cover the high-latitude wind zone to the southwest of the pulsar. Perhaps the knot disrupts the high-latitude wind, leading to the formation of the amorphous and rapidly varying structure of the anvil, while the high-latitude wind zone to the northwest forms the more ordered halo.

\subsection{An Ion-dominated Wind?}

Gallant \& Arons (1994) proposed a detailed model for the Crab Nebula in which the optical wisps are described as inter- 


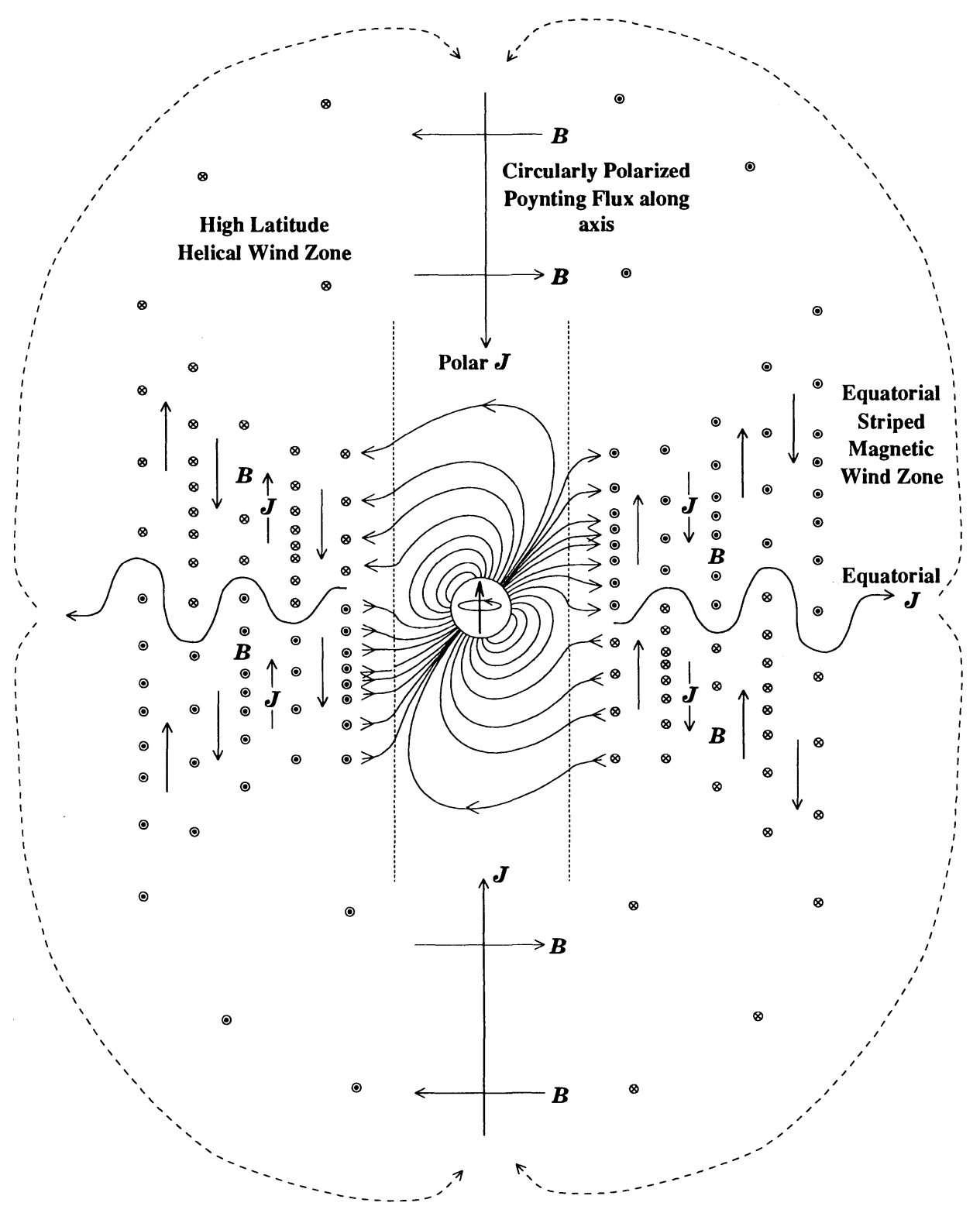

FIG. 16.-Side view of the magnetic field geometry of the wind from an oblique rotator

nal structures within the termination shock of an iondominated pulsar wind. It is not straightforward to compare the new observations with this model in detail; the model was tuned to match the surface brightness profile along the major axis of the Crab as determined from the van den Bergh \& Pritchet (1989) data, and the authors made no detailed predictions about the structure that might be expected at higher resolution. On the other hand, the significant substructure seen within wisp 1 and throughout the vicinity of the X-ray torus may have significant implications for the model.

One very clear comparison between the model and the WFPC 2 data can be made. Gallant \& Arons state that the principal success of the model is its ability to fit the observed separation between wisp 1 and wisp 2 as they existed in 1988 . (The model did not naturally account for part of the structure near the center of the Crab. In particular, it did not account for the thin wisp, a feature seen here to have comparable volume emissivity to wisp 1.) This separation is said to arise from the cyclotron radius of the ions in the flow. However, as discussed above in $\S 3.4$, wisps 2 and 3 were no longer prominent, welldefined features in 1994.

It should also be pointed out that the three-dimensional structure of the Crab suggested here (Fig. 17) is in conflict with the geometry of the Gallant \& Arons model. The Gallant \& Arons model viewed wisp 1 and wisp 2 as lying in the equatorial plane of the pulsar, with wisp 2 located beyond wisp 1 in that plane. In this paper, however, we propose that wisp 1 and the thin wisp are part of a single structure (the halo) that is spatially distinct from the equatorial torus of which wisp 2 was a part. We conclude that both the structure of the wisps as seen 


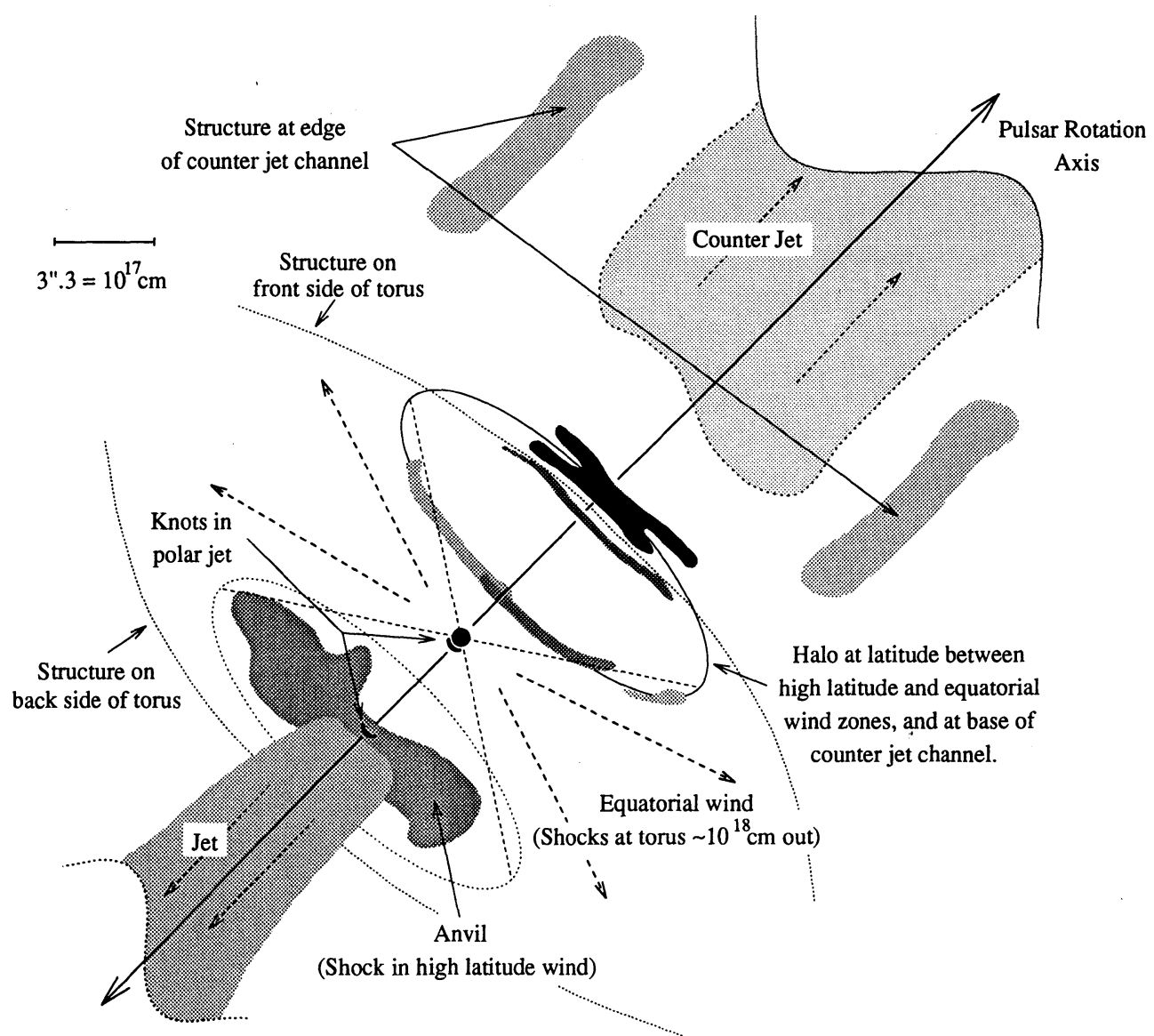

FIG. 17.-Idealized sketch of the proposed structure of the Crab Nebula, including suggested associations between observed features in the nebula and zones found in theoretical models of pulsar winds.

in the WFPC2 data and the geometry that we infer from those data differ significantly from the assumptions made in the Gallant \& Arons model.

\section{SUMMARY}

We have presented a number of new observations of the synchrotron component of the Crab Nebula. These include high spatial resolution images taken with the WFPC2 and $\mathrm{X}$-ray images obtained with the ROSAT HRI. The WFPC2 data resolve much of the structure within the nebula. We report the discovery of a number of previously unknown knots, including two that appear to be persistent over a $5 \mathrm{yr}$ timescale, and that line up with the X-ray/visible jet. One of these knots is located only $1500 \mathrm{AU}$ above the southeast pole of the pulsarthe closest known structure to the pulsar. There does not appear to be a corresponding knot above the other pole of the pulsar, which seems to break the expected symmetry of the wind near the neutron star. It is tentatively suggested that two of the famous "wisps" in the center of the Crab are part of a single halo that circles the symmetry axis of the system. Equipartition fields and pressures in the knots and wisps have been computed from their measured volume emissivities and are found to be much higher than the nebular average. The WFPC2 data also show that a very fine brushstroke or fibrous texture swirls through the Crab, tracing the magnetic structure within the nebula.

The WFPC2 data were compared with a very good CFHT image (van den Bergh \& Pritchet 1989) to explore the temporal variability of structure within the Crab at 0.5 resolution. Despite the considerable improvement in resolution over previous studies, however, we leave the fundamental question of the distinction between "change" and "motion" within the center of the Crab unanswered. In the outer nebula, on the other hand, clear motion of large coherent features is seen, indicating that magnetic structure moves through the nebula at a rate much faster than homologous expansion.

The ROSAT data generally confirm an earlier "torus/jet" description of the X-ray emission from the Crab, but. show considerable new substructure within this bright inner region. They also show newly detected low surface brightness structure in the outer nebula, including $\mathrm{X}$-rays associated with the east and west bays. Much of the X-ray structure of the Crab is found to be associated with features seen in visible emission. Comparison of the WFPC2 data with the X-ray structure of the Crab leads us to suggest that the sharpest of the visible fibers are predominantly localized to a thin region just outside the shock in the pulsar's equatorial wind. An X-ray counterjet to the northwest of the pulsar appears to be surrounded by a channel seen in visible emission.

This wide range of observations has been tied together into a picture of the physical morphology of the center of the Crab that is symmetrical about the (presumed) rotation axis of the pulsar but that shows both pronounced structure as a function of latitude and a significant asymmetry between the two hemi- 
spheres of the system. We have begun the process of tying this observational picture to a theoretical interpretation by suggesting a starting point for associating the observed features in the interior of the Crab with zones found in models of pulsar winds.

As a final note, there remain any number of important outstanding questions - in particular, questions about the shortterm variability of the $\mathrm{Crab}$, particle injection mechanisms over the scales defined by the observed structure, and the details of the magnetic field topology, especially in the newly discovered knot near the pulsar. Answers to these questions await more extensive study of the polarization, spectral index variations, and time evolution of the inner synchrotron nebula at $0^{\prime \prime} .1$ resolution.

We wish to thank S. van den Bergh and C. J. Pritchet, who graciously provided us with a copy of their $I$-band image to allow comparison with the WFPC2 data. J. J. H. and P. S. would like to acknowledge useful discussions with Curt Michel. We thank B. Aschenbach for calling our attention to the fact that some of the aspects of the Crab ROSAT data, including the existence of the X-ray counterjet and the extent of the emission in the outer nebula, have been previously noted in seminars and colloquia by him and coworkers. This research has made use of data obtained through the High Energy Astrophysics Science Archive Research Center Online Service, provided by the NASA Goddard Space Flight Center. This work has made use of the SIMBAD database, operated at CDS, Strasbourg, France. All data reductions and analysis were carried out using software running within the IDL environment. This work was supported by NASA grant NAS 5-1661 to the WF/PC IDT and NASA contract NAS 7-1260 to the WFPC2 IDT. This work was supported at ASU by NASA/ JPL contracts 959289 and 959329 and Caltech contract PC 064528 .

\section{REFERENCES}

Aschenbach, B. 1992, Zeiss Info. Jenaer Rundschau, 1, 6

Aschenbach, B., \& Brinkmann, W. 1975, A\&A, 41, 147

Begelman, M. C., \& Li, Z.-Y. 1992, ApJ, 397, 187

Bietenholz, M. F., \& Kronberg, P. P. 1992, ApJ, 393, 206

Bietenholz, M. F., Kronberg, P. P., Hogg, D. E., \& Wilson, A. S. 1991, ApJ, 373, L59

Bowyer, S., Byram, E. T. Chubb, T. A., \& Friedman, H. 1964, Science, 146, 912

Brinkmann, W., Aschenbach, B., \& Langmeier, A. 1985, Nature, 313, 662

Burrows, C. J., et al. 1994, Wide Field and Planetary Camera 2 Instrument Handbook, ed. C. J. Burrows (Baltimore: STSCI)

Bychkov, K. V. 1975, Soviet Astron--AJ, 18, 420

Cocke, W. J., Disney, M. J., \& Taylor, D. J. 1969, Nature, 221, 525

Cordes, J. M., Romani, R. W., \& Lundgren, S. C. 1993, Nature, 362, 133

Coroniti, F. V. 1990, ApJ, 349, 538

Craig, I. J. D., McClements, K. G., Thompson, A. M., \& Brown, J. C. 1985, A\&A, 149, 171

Davidson, K., \& Fesen, R. A. 1985, AR\&A, 23, 119

Dombrovsky, V. A. 1954, Dokl. Akad. Nauk SSSR, 94, 1021

Fesen, R. A., Martin, C. L., \& Shull, J. M. 1992, ApJ, 399, 599

Gallant, Y. A., \& Arons, J. 1994, ApJ, 435, 230

Goldreich, P., \& Julian, W. H. 1969, ApJ, 157, 869

Gull, T. R., \& Fesen, R. A. 1982, ApJ, 260, L75

Gunn, J. E., \& Ostriker, J. P. 1971, ApJ, 165, 523

Harnden, F. R., Jr., \& Seward, F. D. 1984, ApJ, 283, 279

Henry, R. B. C., \& MacAlpine, G. M. 1982, ApJ, 258, 11

Hester, J. J., Graham, J. R., Beichman, C. A., \& Gautier, T. N., III. 1990, ApJ, 357,539

Hester, J. J., \& Kulkarni, S. R. 1988, ApJ, 331, L121 , 1989, ApJ, 340, 362

Hester, J. J., Scowen, P. A., Gallagher, J. S., \& the WFPC2 IDT. 1994, BAAS, 26,951

Hickson, P., \& van den Bergh, S. 1990, ApJ, 365, 224

Holtzman, J. A., et al. 1994, PASP, in press

Kennel, C. F., \& Coroniti, F. V. 1984a, ApJ, 283, 694

. 1984b, ApJ, 283, 710

Kronberg, P. P., Lesch, H., Ortiz, P. F., \& Bietenholz, M. F. 1993, ApJ, 416, 251
Kulkarni, S. R., \& Hester, J. J. 1988, Nature, 335, 801

Lampland, C. O. 1921, PASP, 33, 79

Lawrence, S. S., MacAlpine, G. M., Uomoto, A., Woodgate, B. E., Brown,

L. W., Oliversen, R. J., Lowenthal, J. D., \& Liu, C. 1994, AJ, submitted

Li, Z.-Y., \& Begelman, M. C. 1992, ApJ, 400, 186

Mauche, C. W. \& Gorenstein, P. 1989, ApJ, 336, 843

Michel, F. C. 1971, Comments Astrophys. Space Phys., 3, 80 1982, Rev. Mod. Phys., 54, 1 1994, ApJ, 432, 239

Michel, F. C., Scowen, P. A., Dufour, R. J., \& Hester, J. J. 1991, ApJ, 368, 463

Nolan, P. L., et al. 1993, ApJ, 409, 697

Oort, J. H., \& Walraven, T. 1956, B.A.N., 12 (462), 285

Pacholczyk, A. G. 1970, Radio Astrophysics (San Francisco: Freeman)

Pearson, T. J., \& Zensus, J. A. 1987, in Superluminal Radio Sources, ed. J. A. Zensus \& T. J. Pearson (Cambridge: Cambridge Univ. Press)

Pelling, R. M., Paciesas, W. S., Peterson, L. E., Makishima, K., Oda, M. Ogawara, Y., \& Miyamoto, S. 1987, ApJ, 319, 416

Péquignot, D., \& Dennefeld, M. 1983, A\&A, 120, 249

Percival, J. W., et al. 1993, ApJ, 407, 276

Piddington, J. H. 1957, Australian J. Phys., 10, 530

Rees, M. J., \& Gunn, J. E. 1974, MNRAS, 167,

Scargle, J. D. 1969, ApJ, 156, 401

Scowen, P. A., Hester, J. J., Gallagher, J. S., Watson, A., Lynds, R., O'Neil, E. J., Jr., \& the WF/PC and WFPC2 IDTs. 1994, BAAS, 26, 951

Seward, F. D. 1981, in Proc. NATO Advanced Study Institute on Supernovae, ed. M. J. Rees \& R. J. Stoneham (Dordrecht: Reidel)

Shklovsky, I. S. 1953, Dokl. Akad. Nauk SSSR, 90, 983

Sulkanen, M. E., \& Lovelace, R. V. E. 1990, ApJ, 350, 732

Trauger, J. T., et al. 1994, ApJ, 435, L3

Trimble, V. L. 1968, AJ, 73, 535

- 1982, Rev. Mod. Phys., 54, 1183

Uomoto, A., \& MacAlpine, G. M. 1987, AJ, 93, 1511

van den Bergh, S. 1970, ApJ, 160, L27

van den Bergh, S., \& Pritchet, C. J. 1989, ApJ, 338, L69

Wilson, D. B., \& Rees, M. J. 1978, MNRAS, 185, 297

Wu, C.-C. 1981, ApJ, 245, 581

Wyckoff, S., \& Murray, C. A. 1977, MNRAS, 180, 717 